\title{
Evolutionary and convergence stability for continuous phenotypes in finite populations derived from two-allele models
}

\author{
Joe Yuichiro Wakano ${ }^{\mathrm{a}, \mathrm{b}, *}$, Laurent Lehmann ${ }^{\mathrm{c}}$ \\ ${ }^{a}$ Meiji Institute for Advanced Study of Mathematical Sciences, Meiji University, Japan \\ ${ }^{\mathrm{b}}$ PRESTO, Japan Science and Technology Agency, Japan \\ ${ }^{\mathrm{c}}$ Department of Ecology and Evolution, University of Lausanne, Switzerland
}

\section{H I G H L I G H T S}

- We propose a stochastic evolutionary model in continuous trait axis.

- The model assumes resident-mutant system with very small mutation rate.

- The model is analyzed by population-genetic methods including moment closure.

- We study the first- and second-order stability conditions.

- Convergence stability alone is enough to characterize long-term evolution.

\section{A R T I C L E I N F O}

\section{Article history:}

Received 12 December 2011

Received in revised form

26 June 2012

Accepted 27 June 2012

Available online 7 July 2012

Keywords:

Fixation probability

Stationary average frequency

Canonical diffusion

Adaptive dynamics

Population genetics

\begin{abstract}
A B S T R A C T
The evolution of a quantitative phenotype is often envisioned as a trait substitution sequence where mutant alleles repeatedly replace resident ones. In infinite populations, the invasion fitness of a mutant in this two-allele representation of the evolutionary process is used to characterize features about longterm phenotypic evolution, such as singular points, convergence stability (established from first-order effects of selection), branching points, and evolutionary stability (established from second-order effects of selection). Here, we try to characterize long-term phenotypic evolution in finite populations from this two-allele representation of the evolutionary process. We construct a stochastic model describing evolutionary dynamics at non-rare mutant allele frequency. We then derive stability conditions based on stationary average mutant frequencies in the presence of vanishing mutation rates. We find that the second-order stability condition obtained from second-order effects of selection is identical to convergence stability. Thus, in two-allele systems in finite populations, convergence stability is enough to characterize long-term evolution under the trait substitution sequence assumption. We perform individual-based simulations to confirm our analytic results.
\end{abstract}

(c) 2012 Elsevier Ltd. All rights reserved.

\section{Introduction}

The evolution of a quantitative phenotype (e.g., antler size, sex-ratio, parental effort into offspring survival) is often envisioned as a step-by-step transformation through the successive invasion of mutant alleles expressing different phenotypic features than those coded by resident alleles. A simple and convenient model is the evolution of a one dimensional phenotype where only two alleles, mutant and resident, compete against each other. Once either mutant or resident wins, another

\footnotetext{
* Corresponding author at: Meiji Institute for Advanced Study of Mathematical Sciences, Meiji University, Japan. Tel.: +81 44934 7675; fax: +81 449347660.

E-mail address: joe@meiji.ac.jp (J. Yuichiro Wakano).
}

mutant is introduced to trigger a new competition. This trait substitution sequence provides a basic framework of classic theories of adaptive dynamics and evolutionary game theory (Maynard-Smith, 1982; Eshel, 1983; Hammerstein, 1996; Metz et al., 1996; Champagnat et al., 2006). Given the complexity of the genetic and demographic processes underlying the trait substitution process in natural populations, to what extent can the end points or the polymorphic states of the evolutionary dynamics be predicated from simple criteria?

The concept of evolutionary stability (Maynard-Smith, 1982; Eshel, 1983) is born out of the aim of characterizing long-term phenotypic evolution directly from payoff functions, devoid of the intricacies of population genetics considerations. The strength of the concept of evolutionary stability has been forcefully revealed when analyzing the evolution of continuously varying phenotypes, 
where it has been applied to investigate the evolution of many of different traits, from the sex-ratio in spatially structured populations, to body size in prey-predator systems, to the evolution of other-regarding preferences (e.g., Parker and Maynard Smith, 1990; Bulmer, 1994; Taylor, 1994; Geritz et al., 1998; Vincent and Brown, 2005; Dercole and Rinaldi, 2008; Leimar, 2009; Akçay and Van Cleve, 2012).

Eshel (1983) identified two questions pertaining to the longterm stability of a continuous strategy. (i) If $z$ is a phenotype expressed by most individuals in a population, will the expected fitness of its carriers be at least as high as the expected fitness to individuals expressing any alternative phenotype, whenever individuals carrying the mutant phenotype are in the minority? This is the question of evolutionary stability. (ii) If a large enough number of individuals in the population express a phenotype $z+\delta$, which is sufficiently close to $z$, will it be advantageous for a mutant individual with deviation $\delta$ in this population to express a phenotype even closer to, rather than farther away from $z$ ? This is the question of convergence stability. Eshel (1983) demonstrated that a positive answer to question (i) does not necessarily imply a positive answer to question (ii).

Although much attention has been paid to theoretical aspects of selection in finite population with and without frequencydependent selection (e.g., Wright, 1931; Crow and Kimura, 1970; Gandon and Rousset, 1999; Ewens, 2004; Rousset and Ronce, 2004; Otto and Day, 2007; Lessard, 2005; Lessard and Ladret, 2007; Tarnita et al., 2009; Ohtsuki, 2010), there are few studies (Rousset and Billiard, 2000; Rousset, 2004) addressing the theoretical question of the stability of continuous phenotypes in finite populations (for discrete strategies, see Nowak et al., 2004; Wild and Taylor, 2004; Lessard, 2005). Here, random genetic drift can markedly increase the frequency of a mutant allele even if it would be always eliminated by selection in a large population. Thus, a superior allele does not always fix in a finite population.

Under the standard trait substitution sequence assumption (two-allele system), there are at least three different ways to determine the evolutionary advantage of a mutant over a resident. First, by determining whether the direct fitness (or invasion fitness) of a rare mutant is larger than that of the resident. Second, by determining whether the mutant has a higher than neutral fixation probability. Finally, by establishing whether the mutant has a higher stationary average frequency than the resident allele in the presence of a low recurrent mutation rate. To the first order in selection intensity, these three ways of determining the evolutionary advantage of the mutant are equivalent (Rousset and Billiard, 2000; Taylor et al., 2007). Hence, singular points and convergence stability conditions for finite populations can be ascertained from direct fitness alone.

However, whether disruptive selection and eventually branching occur at a singular point cannot be determined from considering only first-order effects of selection. Several studies have considered second-order effects to answer the question of evolutionary stability (Eshel, 1983; Taylor, 1989; Lessard, 1990; Geritz et al., 1998). But this has not been much investigated in the context of the evolution of continuous phenotypes in finite populations.

While mutual invasion and the coexistence of different phenotypes are usually discussed in relation to evolutionary branching (e.g., Metz et al., 1996), only a few mathematical analysis have been done in this context from the perspective of stochastic dynamical systems (Champagnat and Méléard, 2011). Further, even within the assumption of the trait substitution sequence, second-order effects on the fixation probabilities and the resulting long-term evolutionary dynamics have not been studied by a stochastic model taking the effect of small population size into account.
The aim of this paper is to investigate second order effects in selection intensity and their connection to long-term evolutionary dynamics within the trait substitution sequence framework. We first recall standard conditions of evolutionary and convergence stability by a deterministic model allowing non-rare mutants. Then we extend this model to finite population size to derive a stochastic model. We analytically derive stability conditions in the stochastic model and discuss their relation to standard conditions. Finally, we apply our results to a pairwise public goods game where evolutionary branching can occur and compare our results with results of individual-based simulations.

\section{Deterministic model}

We consider a haploid population of constant and infinite size. This population is not subdivided into classes (be it by geography, age, or stage) and individuals reproduce at discrete time points. We assume that two alleles segregate in the population. Individuals carrying a mutant allele, denoted by A, express a continuously varying strategy that takes the value $z+\delta$, where $z$ is the phenotypic value expressed by individuals carrying the resident allele, say a. In each generation, individuals interact with each other so that the payoff of an individual depends on its own phenotype as well as on that of others. Let $f\left(z_{1}, z_{2}\right)$ be the payoff given to a player taking strategy $z_{1}$ when matched with a player taking strategy $z_{2}$. We consider that interactions occur at random so that we can write the fecundity (the number of offspring produced) of both types of alleles in the population when the frequency of the mutant is $p_{\mathrm{A}}$ as

$F_{\mathrm{A}}=p_{\mathrm{A}} f(z+\delta, z+\delta)+\left(1-p_{\mathrm{A}}\right) f(z+\delta, z)$

$F_{\mathrm{a}}=p_{\mathrm{A}} f(z, z+\delta)+\left(1-p_{\mathrm{A}}\right) f(z, z)$.

The change in the frequency $p_{\mathrm{A}}$ of the mutant allele over one generation obeys the discrete-time version of replicator equation

$\Delta p_{\mathrm{A}}=p_{\mathrm{A}}\left(1-p_{\mathrm{A}}\right)\left(w_{\mathrm{A}}-w_{\mathrm{a}}\right)$,

where $p_{A}\left(1-p_{A}\right)$ is the variance in gene frequency, $w_{A} \equiv F_{\mathrm{A}} /\left[p_{\mathrm{A}} F_{\mathrm{A}}+\left(1-p_{\mathrm{A}}\right) F_{\mathrm{a}}\right]$ is the fitness of a mutant individual, while $w_{\mathrm{a}} \equiv F_{\mathrm{a}} /\left[p_{\mathrm{A}} F_{\mathrm{A}}+\left(1-p_{\mathrm{A}}\right) F_{\mathrm{a}}\right]$ is the fitness of a resident so that fitness defined in this way gives the total number of descendants of an individual after one full iteration of the life cycle of the organism (Hamilton, 1964, p. 1).

By way of a Taylor expansion of Eq. (2) around $\left(z_{1}, z_{2}\right)=(z, z)$, the allele frequency change can be written as

$\Delta p_{\mathrm{A}}=p_{\mathrm{A}}\left(1-p_{\mathrm{A}}\right)\left[\delta \frac{f_{1}}{f}+\frac{\delta^{2}}{2}\left(\frac{f_{11}+2 p_{\mathrm{A}} f_{12}}{f}-\frac{p_{\mathrm{A}} f_{1}\left(f_{1}+f_{2}\right)}{f^{2}}\right)\right]+O\left(\delta^{3}\right)$,

where $f_{i}$ is the partial derivative of $f(\cdot, \cdot)$ with respect to the $i$-th argument and $f_{i j}$ denotes the function derived once with respect to the $i$-th and a second time with respect to the $j$-th argument (see Eq. (A.2) in Online Supplement). When $\delta$ is small, the change of allele frequency is approximately $\Delta p_{\mathrm{A}} \simeq p_{\mathrm{A}}\left(1-p_{\mathrm{A}}\right) \delta f_{1} / f$ and the direction of evolution is determined by the sign of the selection gradient $f_{1} / f$. Since $f \geq 0$, the direction of evolution is determined by the sign of $f_{1}$. When $f_{1} \neq 0$ holds, selection is unidirectional (the dynamics of $p_{\mathrm{A}}$ is monotonic increasing or decreasing), independently of the value $p_{\mathrm{A}}$, and thus the sign of $f_{1}$ predicts the direction of evolution on the $z$-axis.

At a singular point $z^{*}$ satisfying $f_{1}\left(z^{*}\right)=0$, we have $\Delta p_{\mathrm{A}}=0$ to the first order in selection intensity and the direction of selection is 
determined by the second order term in Eq. (3), which reduces to

$\Delta p_{\mathrm{A}}=p_{\mathrm{A}}\left(1-p_{\mathrm{A}}\right) \frac{\delta^{2}}{2}\left(\frac{f_{11}+2 p_{\mathrm{A}} f_{12}}{f}\right)+O\left(\delta^{3}\right)$.

When the residents adopt a singular strategy, the frequency of mutants taking a strategy $z^{*}+\delta$ decreases to the second order in selection intensity if

$f_{11}\left(z^{*}\right)+2 p_{A} f_{12}\left(z^{*}\right)<0$.

Although this is frequency dependent, the standard condition for evolutionary stability (Maynard-Smith, 1982) is obtained by assuming vanishing mutant frequencies as it is defined from $F_{\mathrm{A}}-F_{\mathrm{a}}$ evaluated at $p_{\mathrm{A}} \rightarrow 0$ (Bishop and Cannings, 1978, p. 90). Hence a singular strategy is evolutionarily stable if $f_{11}\left(z^{*}\right)<0$.

Whether or not a population will eventually reach a singular point $z^{*}$ depends on the condition of convergence stability (Eshel, 1983), which can be characterized from the selection gradient when neither the mutant nor the resident adopts a singular strategy. As $f_{1}$ in Eq. (3) does not vanish and the selection gradient becomes independent of the frequency of the mutant, we reproduce the traditional convergence stability condition

$\left.\frac{d f_{1}(z)}{d z}\right|_{z=z^{*}}=f_{11}\left(z^{*}\right)+f_{12}\left(z^{*}\right)<0$,

under which the allele that has a closer phenotypic value to $z^{*}$ replaces the other allele for any allele frequency $p$.

In summary, we have three standard conditions, one for singular strategy $\left(Q_{S}\right)$, one for evolutionary stability $\left(Q_{\mathrm{ES}}\right)$, and one for convergence stability $\left(Q_{\mathrm{cs}}\right)$

$Q_{\mathrm{S}}: f_{1}\left(z^{*}\right)=0$

$Q_{\mathrm{ES}}: f_{11}\left(z^{*}\right)<0$

$Q_{\mathrm{CS}}:\left.\frac{d f_{1}(z)}{d z}\right|_{z=z^{*}}=f_{11}\left(z^{*}\right)+f_{12}\left(z^{*}\right)<0$

(Eshel, 1983; Taylor, 1989; Lessard, 1990; Geritz et al., 1998).

\section{Stochastic model}

\subsection{Allele frequency change}

\subsubsection{Formulation}

We now reformulate the previous model and consider that the population is of constant but finite size $N$. There are at least two different ways to study long-term evolution in finite populations. First, calculating a fixation probability in a model without mutations (Crow and Kimura, 1970). Second, the long-term expected frequency of the mutant under a selection-drift-recurrent mutation process (Crow and Kimura, 1970). Hence, it is natural to try to study evolutionary stability either from the fixation probability of the mutant or from its long-term frequency in the presence of mutation. In the latter approach, we assume that the mutation rate and step size is so small that the expected time until a new mutant appears is much longer than the expected time until competition between two alleles leads to the fixation of one of them (separation of time scales assumption, Gillespie, 1991; Metz et al., 1996; Champagnat et al., 2006). By assuming this vanishing mutation rate, we can remain in the mutant-resident framework, or the two-allele system (Rousset and Billiard, 2000; Taylor et al., 2007).

Let $\rho_{\mathrm{A}, t}$ be the expected frequency of the mutant allele at time $t$. Call $\pi_{\mathrm{A}}\left(N_{\mathrm{A}}\right)$ the fixation probability of the mutant in a population with an initial number $N_{\mathrm{A}}$ of mutants and in an evolutionary model without mutations. Then $\pi_{\mathrm{A}}=\lim _{t \rightarrow \infty} \rho_{\mathrm{A}, t}$ holds because the mutant frequency is either zero or one if $t$ is larger than fixation time. We can also calculate stationary average frequency from $\pi_{\mathrm{A}}$ as

$\rho_{\mathrm{A}}=\frac{\pi_{\mathrm{A}}(1)}{\pi_{\mathrm{A}}(1)+\left[1-\pi_{\mathrm{A}}(N-1)\right]}$,

because, under our assumption of vanishing mutation rate, the system almost always stays at either of the two fixation states (Gillespie, 1991; Rousset and Billiard, 2000; Fudenberg and Imhof, 2006).

We now develop the stochastic analog of the replicator equation (Eq. (2)) and write down recursions for the average mutant allele frequency over one generation. As before, we assume that the resident allele takes phenotype $z$ and the mutant allele phenotype $z+\delta$. But by contrast to the previous model, where fitness was defined in an allele-centered way, we now construct the model from an individual-centered perspective. We denote the fitness of individual $i$ by $w_{i}(\mathbf{z})$, which may depend on the phenotypes of the different individuals in the populations. These phenotypes are collected into the vector $\mathbf{z} \equiv\left(z_{1}, \ldots, z_{N}\right)$. The phenotype $z_{i}$ of individual $i$ is written as $z_{i}=z+\delta p_{i}$, where $p_{i}$ takes the value one if individual $i$ carries the mutant, and zero otherwise $\left(p_{i} \in\{0,1\}\right)$.

We denote by $p_{\mathrm{A}} \equiv \sum_{i=1}^{N} p_{i} / N$ the frequency of the mutant in the population, which is determined by the realized values of mutant frequencies in the population that are collected in the vector $\mathbf{p} \equiv\left(p_{1}, \ldots, p_{N}\right)$. The probability distribution of $\mathbf{p}$ at time $t$ which depends on the effect of the mutant $(\delta)$, is denoted by $\operatorname{Pr}(\mathbf{p}(t)=\mathbf{p} ; \delta)$. In other words, we add the symbol $(t)$ to represent a random variable. The expectation of the frequency in the next generation, conditional on the current realization $\mathbf{p}(t)=\mathbf{p}$, can be written as

$\mathrm{E}\left[p_{\mathrm{A}}(t+1) \mid \mathbf{p}(t)=\mathbf{p}\right]=\frac{1}{N} \sum_{i=1}^{N} w_{i}(\mathbf{z}(\mathbf{p})) p_{i}$,

where $w_{i}(\mathbf{z}(\mathbf{p}))$ denotes the fitness of individual $i$ under realization p. This equation sums up the expected numbers of offspring (=fitness) produced by all mutants $\left(p_{i}=1\right)$ while the offspring of residents $\left(p_{i}=0\right)$ are not counted.

We now Taylor expand the fitness function around $\delta=0$ as

$w_{i}(\mathbf{z}(\mathbf{p}))=1+\delta \sum_{j=1}^{N} \frac{\partial w_{i}(\mathbf{z}(\mathbf{p}))}{\partial z_{j}} p_{j}+\frac{\delta^{2}}{2} \sum_{j=1}^{N} \sum_{k=1}^{N} \frac{\partial^{2} w_{i}(\mathbf{z}(\mathbf{p}))}{\partial z_{j} \partial z_{k}} p_{j} p_{k}+O\left(\delta^{3}\right)$,

all partial derivatives are evaluated at the resident phenotypic values $\mathbf{z}=(z, z, \ldots, z)$ corresponding to $\delta=0$. Since we assume a panmictic population, the phenotype of individual $j$ has the same effect on the fitness of any individual $i \neq j$, while having a different effect on itself. Formally, this amounts to the assumption that the following symmetries hold for any $i, j, k, l$

$$
\begin{aligned}
& w_{i, i}=w_{j, j}, \\
& w_{i, i i}=w_{j, j j}, \\
& w_{i, j}=w_{k, l} \quad(i \neq j, k \neq l), \\
& w_{i, i j}=w_{k, k l} \quad(i \neq j, k \neq l), \\
& w_{i, j j}=w_{k, l l} \quad(i \neq j, k \neq l),
\end{aligned}
$$

where

$w_{i, j} \equiv \frac{\partial w_{i}}{\partial z_{j}}, \quad w_{i, j k} \equiv \frac{\partial^{2} w_{i}}{\partial z_{j} \partial z_{k}}$,

which are functions of the phenotype vector, i.e., $w_{i, j}=w_{i, j}(\mathbf{z})$ and $w_{i, j k}=w_{i, j k}(\mathbf{z})$. 


\subsubsection{Connection to the deterministic model}

How is this individual-centered notation related to the deterministic model in the preceding section? The (direct) fitness function of individual $i$ carrying a mutant allele is given by

$w_{i}(\mathbf{z}(\mathbf{p}))=1+\delta w_{i, i} p_{i}+\frac{\delta^{2}}{2} w_{i, i i} p_{i}+($ Effects from other mutants $)+O\left(\delta^{3}\right)$,

where the fourth term represents all mutant-mutant interactions $\left(p_{j} p_{k}\right.$ terms where $j \neq k$ in Eq. (10)). In the traditional definition of ESS (Maynard-Smith, 1982), mutants are assumed to be rare so the fourth term is neglected. By doing so, Eq. (13) shows that the selection gradient on a rare mutant is given by $w_{i, i}$ to the first order. A singular point is given by $w_{i, i}\left(z^{*}\right)=0$ and it is stable by convergence if $d w_{i, i}(z) / d z<0$. At a singular point, the one generational change in allele frequency for a rare mutant is predicted by the sign of $w_{i, i i}$ and thus $z^{*}$ is evolutionary stable if $w_{i, i i}<0$. Hence, taking into account first and second order effects in selection intensity, we have the following stability conditions in a finite population by imposing that the mutant remains rare:

$Q_{S}: w_{i, i}\left(z^{*}\right)=0$,

$Q_{\mathrm{ES}}: w_{i, i i}\left(z^{*}\right)<0$,

$Q_{\mathrm{CS}}:\left.\frac{d w_{i, i}(z)}{d z}\right|_{z=z^{*}}=w_{i, i i}\left(z^{*}\right)+(N-1) w_{i, i j}\left(z^{*}\right)<0$.

This result parallels that for the deterministic model (Eq. (7)), but here we do not assume pairwise interactions and individuals might be playing against the field (Maynard-Smith, 1982).

\subsubsection{Back to the stochastic model}

We now study the evolutionary dynamics when mutants are no longer rare so that mutant-mutant interactions cannot be neglected. We thus return to the evaluation of the fixation probability and the stationary average frequency of the mutant, which capture such interactions. In order to simplify the evaluation of Eq. (9), we will further use the variables

$p_{\mathrm{A} / \mathrm{A}} \equiv \frac{1}{N(N-1)} \sum_{i} \sum_{j \neq i} p_{i} p_{j}$

$p_{\mathrm{A} / \mathrm{A} / \mathrm{A}} \equiv \frac{1}{N(N-1)(N-2)} \sum_{i} \sum_{j \neq i} \sum_{k \neq j, \neq i} p_{i} p_{j} p_{k}$,

where $p_{\mathrm{A} / \mathrm{A}}(t)\left(p_{\mathrm{A} / \mathrm{A} / \mathrm{A}}(t)\right)$ can be interpreted as the probability that two (three) randomly sampled individuals without replacement from the population carry the mutant allele at time $t$.

Substituting the fitness function (Eq. (10)) into Eq. (9), using Eqs. (11) and (15) and the zero-sum property of the partial derivatives (i.e., $\quad \sum_{j=1}^{N} w_{i, j}=\sum_{j=1}^{N} \sum_{k=1}^{N} w_{i, j k}=0$, Rousset, 2004), and rearranging produces

$$
\begin{aligned}
\mathrm{E}\left[p_{\mathrm{A}}(t+1) \mid \mathbf{p}(t)=\right. & \mathbf{p}]=p_{\mathrm{A}}+\left(\delta \dot{a}_{11}+\frac{\delta^{2}}{2} \ddot{a}_{11}\right) p_{\mathrm{A}}+\left(\delta \dot{a}_{12}+\frac{\delta^{2}}{2} \ddot{a}_{12}\right) p_{\mathrm{A} / \mathrm{A}} \\
& +\frac{\delta^{2}}{2} \ddot{a}_{13} p_{\mathrm{A} / \mathrm{A} / \mathrm{A}}+O\left(\delta^{3}\right),
\end{aligned}
$$

where the $\dot{a}_{i j}$ 's ( $\ddot{a}_{i j}$ 's) are first (second) order selection coefficients (Kirkpatrick et al., 2002), which are given by

$\dot{a}_{11}=w_{i, i}$,
$\dot{a}_{12}=-\dot{a}_{11}$,
$\ddot{a}_{11}=w_{i, i i}$,
$\ddot{a}_{12}=2(N-1) w_{i, i j}+(N-1) w_{i, j j}$, $\ddot{a}_{13}=-\ddot{a}_{11}-\ddot{a}_{12}$.

Note that the summation in Eq. (9) is absorbed into Eq. (15) and thus does not appear as a sum over actor specific selection coefficients in Eq. (16). This stems from the fact that we assumed a non-structured population, where the phenotype of individual $j$ has the same effect on the fitness of any individual $i \neq j$, while having a different effect on itself (Eq. (11)).

Eq. (16) can be interpreted as the extension of the replicator equation (Eq. (2)) to finite populations but this is no longer a recurrence equation in a deterministic variable because it gives the expected allele frequency in a descendant generation conditional on the realization $\mathbf{p}(t)=\mathbf{p}$ in the parental generation. However, integrating Eq. (16) over $\operatorname{Pr}(\mathbf{p}(t)=\mathbf{p} ; \delta)$ produces an equation for the unconditional expected mutant allele frequency at time $t$

$\rho_{\mathrm{A}, t+1} \equiv \mathrm{E}\left[p_{\mathrm{A}}(t+1)\right]=\sum_{\mathbf{p}} \mathrm{E}\left[p_{\mathrm{A}}(t+1) \mid \mathbf{p}(t)=\mathbf{p}\right] \operatorname{Pr}(\mathbf{p}(t)=\mathbf{p} ; \delta)$.

According to Eq. (16), we do not need to know the full distribution $\operatorname{Pr}(\mathbf{p}(t)=\mathbf{p} ; \delta)$ to evaluate the expected allele frequency, but only the first three moments at time $t$ are enough to that aim since

$$
\begin{aligned}
\rho_{\mathrm{A}, t+1}= & \rho_{\mathrm{A}, t}+\left(\delta \dot{a}_{11}+\frac{\delta^{2}}{2} \ddot{a}_{11}\right) \rho_{\mathrm{A}, t}+\left(\delta \dot{a}_{12}+\frac{\delta^{2}}{2} \ddot{a}_{12}\right) \rho_{\mathrm{A} / \mathrm{A}, t} \\
& +\frac{\delta^{2}}{2} \ddot{a}_{13} \rho_{\mathrm{A} / \mathrm{A} / \mathrm{A}, t}+O\left(\delta^{3}\right),
\end{aligned}
$$

which depends on the three moments; $\rho_{\mathrm{A}, t}, \rho_{\mathrm{A} / \mathrm{A}, t} \equiv \mathrm{E}\left[p_{\mathrm{A} / \mathrm{A}}(t)\right]$ and $\rho_{\mathrm{A} / \mathrm{A} / \mathrm{A}, t} \equiv \mathrm{E}\left[p_{\mathrm{A} / \mathrm{A} / \mathrm{A}}(t)\right]$.

\subsection{Moment closure}

In order to evaluate explicitly the dynamics of $\rho_{\mathrm{A}, t}$, we use a population genetic moment closure method based on a Markov chain approach (Lehmann and Rousset, 2009). In general, $\rho_{\mathrm{A}}, \rho_{\mathrm{A} / \mathrm{A}}$, and $\rho_{\mathrm{A} / \mathrm{A} / \mathrm{A}}$ all depend on selection. But since we want to evaluate $\rho_{\mathrm{A}}$ only to the second order around $\delta=0$, we can close the system with the variable $\rho_{\mathrm{A} / \mathrm{A}}$ and $\rho_{\mathrm{A} / \mathrm{A} / \mathrm{A}}$ since they are, respectively, multiplied by first and second order selection coefficients (Eq. (19)). It is then sufficient to evaluate $\rho_{\mathrm{A} / \mathrm{A}}$ to the first order of selection and $\rho_{\mathrm{A} / \mathrm{A} / \mathrm{A}}$ only under neutrality $(\delta=0)$, as any higher order effect of selection on $\rho_{\mathrm{A} / \mathrm{A}}$ and $\rho_{\mathrm{A} / \mathrm{A} / \mathrm{A}}$ would result in higher than second order effects of selection on allele frequency change, which are neglected by assumption. Further, under neutrality $\rho_{\mathrm{A} / \mathrm{A} / \mathrm{A}}$ depends on at most a three gene position. In order to evaluate $\rho_{\mathrm{A}}$ to the second order, we then define $\rho_{t} \equiv$ $\left(\rho_{\mathrm{A}, t}, \rho_{\mathrm{A} / \mathrm{A}, t}, \rho_{\mathrm{A} / \mathrm{A} / \mathrm{A}, t}\right)^{\mathrm{T}}$ as the vector of moments at time $t$, and construct the recursion

$\boldsymbol{\rho}_{t+1}=\mathbf{A}(\delta) \boldsymbol{\rho}_{t}$,

with

$\mathbf{A}(\delta)=\mathbf{A}^{\circ}+\delta \dot{\mathbf{A}}+\frac{\delta^{2}}{2} \ddot{\mathbf{A}}+O\left(\delta^{3}\right)$,

where, generically for the above assumptions, we have

$\mathbf{A}^{\circ} \equiv\left(\begin{array}{ccc}1 & 0 & 0 \\ a_{21}^{\circ} & 1-a_{21}^{\circ} & 0 \\ a_{31}^{\circ} & a_{32}^{\circ} & 1-a_{31}^{\circ}-a_{32}^{\circ}\end{array}\right)$,

which is a matrix gathering zero-th order coefficient. Each component $a_{i j}^{\circ}$ can be derived algebraically (see Online Supplement) or by considering it as the probability that a set of genes sampled in $i$ different individuals descend from $j$ different individuals in the 
previous generation (see Section 3.3). We also have

$\dot{\mathbf{A}} \equiv\left(\begin{array}{ccc}\dot{a}_{11} & -\dot{a}_{11} & 0 \\ \dot{a}_{21} & \dot{a}_{22} & -\dot{a}_{21}-\dot{a}_{22} \\ 0 & 0 & 0\end{array}\right)$

and

$\ddot{\mathbf{A}} \equiv\left(\begin{array}{ccc}\ddot{a}_{11} & \ddot{a}_{12} & -\ddot{a}_{11}-\ddot{a}_{12} \\ 0 & 0 & 0 \\ 0 & 0 & 0\end{array}\right)$,

which are matrices gathering first and second order selection coefficients, respectively.

Recall that $\pi_{\mathrm{A}}\left(N_{\mathrm{A}}\right)$ is the fixation probability of the mutant allele in a population consisting initially of $N_{\mathrm{A}}$ mutants. From Eq. (20), we have

$\lim _{t \rightarrow \infty} \mathbf{A}(\delta)^{t} \boldsymbol{\rho}_{0}=\left(\begin{array}{l}\pi_{\mathrm{A}}\left(N_{\mathrm{A}}\right) \\ \pi_{\mathrm{A}}\left(N_{\mathrm{A}}\right) \\ \pi_{\mathrm{A}}\left(N_{\mathrm{A}}\right)\end{array}\right)$,

where the initial moments are given by

$\boldsymbol{\rho}_{0}=\left(\begin{array}{c}N_{\mathrm{A}} / N \\ N_{\mathrm{A}}\left(N_{\mathrm{A}}-1\right) /[N(N-1)] \\ \left.N_{\mathrm{A}}\left(N_{\mathrm{A}}-1\right)\left(N_{\mathrm{A}}-2\right) /[N(N-1)(N-2)]\right)\end{array}\right)$.

Since Eq. (20) is a three-dimensional linear recursion, we can directly calculate the solution in terms of the series in $\delta$. It is clear that $\mathbf{A}^{\circ}$ plays an important role in the asymptotic behavior of mutant frequency (Eq. (25)). As we can see from its second and third row elements, the transition matrix $\mathbf{A}^{\circ}$ is not simple (Eq. (22)). These elements represent the dynamics of the moments in the neutral process and they depend on what kind of life-cycle is assumed for the model (see below for examples). More generally, our construction should apply to any haploid reproductive scheme where the one generation conditional change in allele frequency can be expressed in terms of a single fitness function $w_{i}(\mathbf{z}(\mathbf{p}))$ (Eq. (10)) with evolution occurring in a panmictic population (Eq. (11)). These are the reproductive schemes following into the domain of Kingman's coalescent for constant population size (Donnelly and Tavaré, 1995), but where population size needs not to be very large (see Lessard, 2011 for an application of the perturbation expansions approach in this domain to games with pure strategies).

\subsection{Example: Wright-Fisher and Moran models}

To illustrate the biological meaning of $\mathbf{A}^{\circ}$, let us consider an explicit representation of the coefficients appearing in Eqs. (20)-(24) in terms of demographic parameters for Wright-Fisher and Moran processes (Ewens, 2004). For the Wright-Fisher process without selection, it follows that:

$\mathrm{E}\left[p_{A / A}(t+1) \mid \mathbf{p}(t)=\mathbf{p}\right]=\frac{1}{N} p_{\mathrm{A}}+\frac{N-1}{N} p_{A / A}$,

because two (different) individuals have the same parent with probability $1 / N$, in which case their common parent carries the mutant allele with probability $p_{\mathrm{A}}$. With the remaining probability $(N-1) / N$, they have different parents both of who have the mutant allele with probability $p_{A / A}$. Thus we have $a_{21}^{\circ}=1 / N$. Note that we do not need further information (e.g., $p_{A / A / A}$ ) to determine the dynamics of the second moment.

The result $\left(a^{\circ}{ }_{21}=1 / N\right)$ can also be obtained more algebraically (see Online Supplement), and we derive the coefficients of $\mathbf{A}^{\circ}$ which is summarized in Table 1 , which shows the difference between the Wright-Fisher and Moran processes (Ewens, 2004).
Table 1

Coefficients of $\mathbf{A}^{\circ}$.

\begin{tabular}{lll}
\hline Coefficient & Wright-Fisher & Moran \\
\hline$a^{\circ}{ }_{21}$ & $\frac{1}{N}$ & $\frac{2}{N^{2}}$ \\
$a^{\circ}{ }_{31}$ & $\frac{1}{N^{2}}$ & 0 \\
$a_{32}^{\circ}$ & $\frac{3(N-1)}{N^{2}}$ & $\frac{6}{N^{2}}$ \\
\hline
\end{tabular}

Using Eq. (17) and the results of Online Supplement (Eqs. (A.26) and (A.14)), the second order selection coefficients necessary to evaluate $\dot{A}$ are given for both the Wright-Fisher and the Moran process by

$\dot{a}_{21}=\frac{2}{N} w_{i, i}$

$\dot{a}_{22}=\frac{2(N-3)}{N} w_{i, i}$

and such coefficients for the more general Cannings process are given in Online Supplement (Eqs. (A.26) and (A.32)).

\section{Stability conditions from asymptotic frequencies}

Having derived moment equations, we can now obtain the mutant fixation probability and its stationary average frequency.

\subsection{Fixation probabilities}

The fixation probability of a single mutant allele can be expressed as

$\pi_{\mathrm{A}}(1)=\frac{1}{N}+\delta \dot{\pi}(z)+\frac{\delta^{2}}{2} \ddot{\pi}(z)+O\left(\delta^{3}\right)$

(see Eq. (A.35) of Online Supplement). The zero-th order term is $1 / N$ because this is the fixation probability of the mutant under neutrality. The first-order term is

$\dot{\pi}(z)=\frac{T_{2}}{N} w_{i, i}$,

where $T_{2}=1 / a^{\circ}{ }_{21}$ can be considered as the coalescence time into a common ancestor of two genes in the neutral process. The first order in selection intensity $\dot{\pi}(z)>0$ implies that $\pi_{A}(1)>1 / N$ for $\delta>0$, which means that a single mutant has a higher than neutral fixation probability (Eq. (A.36) of Online Supplement or Rousset and Billiard, 2000). Hence, evolution on the $z$-axis tends to lead to the increase in $z$ value under a trait substitution process. The value of $z^{*}$ satisfying $\dot{\pi}\left(z^{*}\right)=0$ defines a singular strategy; that is, a point where first order effects of selection vanish.

When residents adopt a singular strategy $z^{*}$, whether a mutant has a higher or lower than neutral fixation probability is given by the second order effect, $\ddot{\pi}\left(z^{*}\right)$. From Eq. (11) and Eq. (A.35), we have

$\ddot{\pi}\left(z^{*}\right)=\frac{T_{2}}{N}\left[g_{1} w_{i, i i}+\left(g_{1}-1\right)\left\{2(N-1) w_{i, i j}+(N-1) w_{i, j j}\right\}\right]$,

where $g_{1}=T_{3} / T_{2}$ and $T_{3}=\left(a_{21}^{\circ}+a_{32}^{\circ}\right) /\left(a_{21}^{\circ}\left\{a^{\circ}{ }_{31}+a^{\circ}{ }_{32}\right\}\right)$ can be considered as the coalescence time of three genes into a common ancestor in the neutral process. The sign of $\ddot{\pi}\left(z^{*}\right)$ depends on $g_{1}$ and using the elements in Table 1 , we have

$g_{1}= \begin{cases}\frac{4 N-3}{3 N-2} & \text { Wright-Fisher } \\ \frac{4}{3} & \text { Moran, }\end{cases}$ 
which shows the effect of underlying life cycle assumptions (e.g., Wright-Fisher vs. Moran).

The expression for $\ddot{\pi}\left(z^{*}\right)$ is one of our primary results. The condition $\ddot{\pi}\left(z^{*}\right)<0$ implies that $\pi_{\mathrm{A}}(1)<1 / N$, so it captures the stability of a singular strategy to the second-order effects in a single event of invasion of a mutant. However, $\ddot{\pi}\left(z^{*}\right)<0$ does not imply that a single resident allele has a higher than neutral fixation probability (Eq. (A.38)). Hence, under the trait substitution sequence assumption, the sign of $\ddot{\pi}\left(z^{*}\right)$ is not indicative of the direction of evolution on the $z$-axis in the presence of mutations, and it cannot be used here to characterize long-term phenotypic evolution. To that aim, we now turn to the stationary average mutant frequency.

\subsection{Stationary average frequency}

\subsubsection{First and second order effects}

We find that we can express the stationary average frequency of the mutant allele in the presence of vanishing mutation rates as

$\rho_{\mathrm{A}}=\frac{1}{2}+\delta \dot{\rho}(z)+\frac{\delta^{2}}{2} \ddot{\rho}(z)+O\left(\delta^{3}\right)$

(see Eq. (A.39) of Online Supplement). The zero-th order term is one half because the two alleles have the same frequencies on average in the absence of selection. Any increase over one half is indicative of positive selection on the mutant allele with all possible realizations of allele frequencies in the population taken into consideration.

The first-order term is

$\dot{\rho}(z)=\frac{L_{2}}{4} w_{i, i}$,

where $L_{j}>0$ can be considered as the total branch length in number of generations of a coalescent tree involving $i$ genes in the neutral process (Eq. (A.40)). For $\dot{\rho}(z)>0$ and $\delta>0, \rho_{\mathrm{A}}>1 / 2$ holds to the first-order. In addition, the condition $\dot{\rho}(z)>0$ is equivalent to $\dot{\pi}(z)>0$. This means that the competition between the mutant and the resident tends to favor the former and that evolution on the $z$-axis tends to increase the value of $z$ in the trait substitution sequence process.

At a singular point $z^{*}$, a mutant allele is selected against to the second-order effects if

$\ddot{\rho}\left(z^{*}\right)=\frac{L_{2}}{4}\left[g_{2} w_{i, i i}+\left(g_{2}-1\right)\left\{2(N-1) w_{i, i j}+(N-1) w_{i, j j}\right\}\right]<0$,

where $g_{2}=L_{3} / L_{2}$ (Eqs. (A.34)-(A.39) in Online Supplement). Using the elements in Table 1, Eqs. (A.34) and (A.39), we can directly calculate

$g_{2}=\frac{3}{2}$

for both the Wright-Fisher and Moran processes. More generally, an argument based on coalescence probabilities shows that this value holds whenever matrix $\mathbf{A}^{\circ}$ (Eq. (22)) describes the dynamics of moments of allele frequencies. That is, it holds for any biological system whose neutral process can be described by Eq. (22) (Eqs. (A.41)-(A.42) in Online Supplement).

We denote the condition $\ddot{\rho}\left(z^{*}\right)<0$ as $Q_{\mathrm{TS}}$ ("two-allele stability") in order to emphasize that it is obtained by considering only two alleles at a time in the population. It is a stochastic analogue of the evolutionary stability condition because second-order effects in selection intensity are taken into account. Further, by contrast to that of $\ddot{\pi}\left(z^{*}\right)$, the sign of $\ddot{\rho}\left(z^{*}\right)$ does not depend on the underlying life-cycle assumption, which is an additional reason to define a two-allele stability condition based on $\ddot{\rho}\left(z^{*}\right)$.

Whether or not evolution will tend to the singular point depends on whether $z^{*}$ is convergence stable. The condition is that $\dot{\rho}(z)$ is a decreasing function at $z=z^{*}$, i.e., $d \dot{\rho}(z) / d z<0$, and from Eqs. (11) and (34), yields a CS condition obtained from the perspective of stationary average frequencies

$\frac{d w_{i, i}(z, z, \ldots, z)}{d z}=\sum_{j}^{N} w_{i, i j}=w_{i, i i}+(N-1) w_{i, i j}<0$.

Thus, using $g_{2}=3 / 2$ into Eq. (35), we obtain the following stability conditions for evolution on the $z$-axis

$Q_{\mathrm{S}}: w_{i, i}\left(z^{*}\right)=0$,

$Q_{\mathrm{TS}}: 3 w_{i, i i}+2(N-1) w_{i, i j}+(N-1) w_{i, j j}<0$,

$Q_{\mathrm{CS}}:\left.\frac{d w_{i, i}(z)}{d z}\right|_{z=z^{*}}=w_{i, i i}\left(z^{*}\right)+(N-1) w_{i, j j}\left(z^{*}\right)<0$.

If $Q_{\mathrm{Ts}}$ and $Q_{\mathrm{CS}}$ are of opposite signs at a given singular point $z^{*}$, then first-order effects in selection intensity tend to push the system towards (away from) $z^{*}$, while second-order effects tend to push it away from (towards) $z^{*}$. In a two-allele system, one then expects the first-order effects to dominate and it may be intuitively felt that $Q_{\mathrm{TS}}$ and $Q_{\mathrm{CS}}$ cannot be of opposite signs, which we now demonstrate.

4.2.2. Two-allele stability reduces to convergence stability We have

$\ddot{\rho}\left(z^{*}\right)<0 \Leftrightarrow 3 w_{i, i i}+2(N-1) w_{i, i j}+(N-1) w_{i, j j}<0$

and using

$w_{i, i i}+(N-1) w_{i, j j}=w_{i, i i}+(N-1) w_{j, i i}=\frac{\partial^{2}}{\partial z_{i}^{2}}\left(\sum_{j=1}^{N} w_{j}\right)=0$,

where we used the symmetry assumptions (Eq. (11)) and the fact that the fitness functions always sum up to $N$, we have

$\ddot{\rho}\left(z^{*}\right)<0 \Leftrightarrow w_{i, i i}+(N-1) w_{i, i j}<0$.

Thus, as long as $g_{2}=3 / 2$, the CS and TS conditions are identical

$d \dot{\rho}\left(z^{*}\right) / d z<0 \Leftrightarrow \ddot{\rho}\left(z^{*}\right)<0$.

In summary, by taking into account second order effects in selection intensity, we arrive at the two conditions

$Q_{S}: w_{i, i}\left(z^{*}\right)=0$,

$Q_{\mathrm{CS}}:\left.\frac{d w_{i, i}(z)}{d z}\right|_{z=z^{*}}=w_{i, i i}\left(z^{*}\right)+(N-1) w_{i, j j}\left(z^{*}\right)<0$,

which characterize long-term phenotypic evolution on the $z$-axis under the trait substitution sequence assumption (two-allele system).

\subsection{Stationary phenotypic distribution}

We have considered only a two-allele system, from which we inferred long term evolution on the $z$-axis (Eq. (43)). Here, we show that this result is consistent with that obtained from a stochastic model allowing for a continuum of alleles under the limit of small mutation rates. Call $\psi(z)$ the probability density function that phenotype $z$ obtains in the population at a mutation-selection-drift balance. Under the assumption of small mutation step size ( $\delta$ small), this probability density function is given by

$\psi(z)=C \exp \left[\int^{z} 2 N \dot{\pi}(y) \mathrm{d} y\right]$

where is $C$ is a normalizing constant (for derivation, see Eqs. (A.45)-(A.52) in Online Supplement). This is the stationary solution of the so-called canonical diffusion of adaptive dynamics, which itself reduces to the canonical equation of adaptive 
dynamics in the limit of very large population size (Champagnat and Lambert, 2007).

The maxima of $\psi(z)$ are the most likely phenotypic outcomes of evolution under the mutation-selection-drift balance. Hence, a population of residents that is in small neighborhood from a local or global optimum of $\psi(z)$ is likely to be replaced by a population of mutants that expresses a phenotypic value closer to that point. Conversely, if a population is located at a minimum of $\psi(z)$, then a mutant that expresses a phenotypic value away from that of the resident is likely to invade. Hence, an internal singular point $z^{*}$ can be said to be stable by convergence if $\mathrm{d} \psi(z) / \mathrm{d} z=\left.0\right|_{z=z^{*}}$ and $\mathrm{d}^{2} \psi(z) / \mathrm{d} z^{2}<\left.0\right|_{z=z^{*}}$ (Lehmann, 2012). This condition reproduces our $Q_{s}$ and $Q_{c s}$ conditions (Eq. (43)), which corroborates our result that convergence stability alone carries sufficient information to predict long-term evolutionary dynamics under the traits substitution sequence assumption, even when second order effects in selection intensity are taken into account.

\section{Applications}

In order to check the accuracy of our approximations and in order to obtain some insights on the conditions under which long-term evolution can be well approximated by a two alleles system, we analyze a specific example of the evolution of continuous phenotype in a non-linear cooperator/defector game, where branching can occur in a large population.

\subsection{Pairwise interaction game}

In our analysis in Sections 3 and 4, we did not make any assumption on whether individuals were playing the field or were randomly matched in pairwise interactions, as is standardly assumed in models defining stability conditions (MaynardSmith, 1982; Bishop and Cannings, 1978; Weibull, 1997). In order to provide an application of our results, we now consider that fitness depends on pairwise interactions and that the interaction between individual $i$ and $j$ leads to payoff $f\left(z_{i}, z_{j}\right)$ to individual $i$.

We assume that individual $i$ interacts with all other individuals $j(\neq i)$ with the same probability so that its fecundity is given by the average payoff

$F_{i}(\mathbf{z})=\frac{1}{N-1} \sum_{j \neq i}^{N} f\left(z_{i}, z_{j}\right)$,

and the population-average fecundity is $\bar{F}(\mathbf{z})=\sum_{i} F_{i}(\mathbf{z}) / N$, which gives the fitness of individual $i$ as

$W_{i}(\mathbf{z})= \begin{cases}\frac{F_{i}(\mathbf{z})}{\bar{F}(\mathbf{z})} & \text { Wright-Fisher } \\ \frac{N-1}{N}+\frac{1}{N} \frac{F_{i}(\mathbf{z})}{\bar{F}(\mathbf{z})} & \text { Moran. }\end{cases}$

Substituting the fitness functions (Eqs. (45) and (46)) into the stability conditions, they can be expressed in terms of fecundity
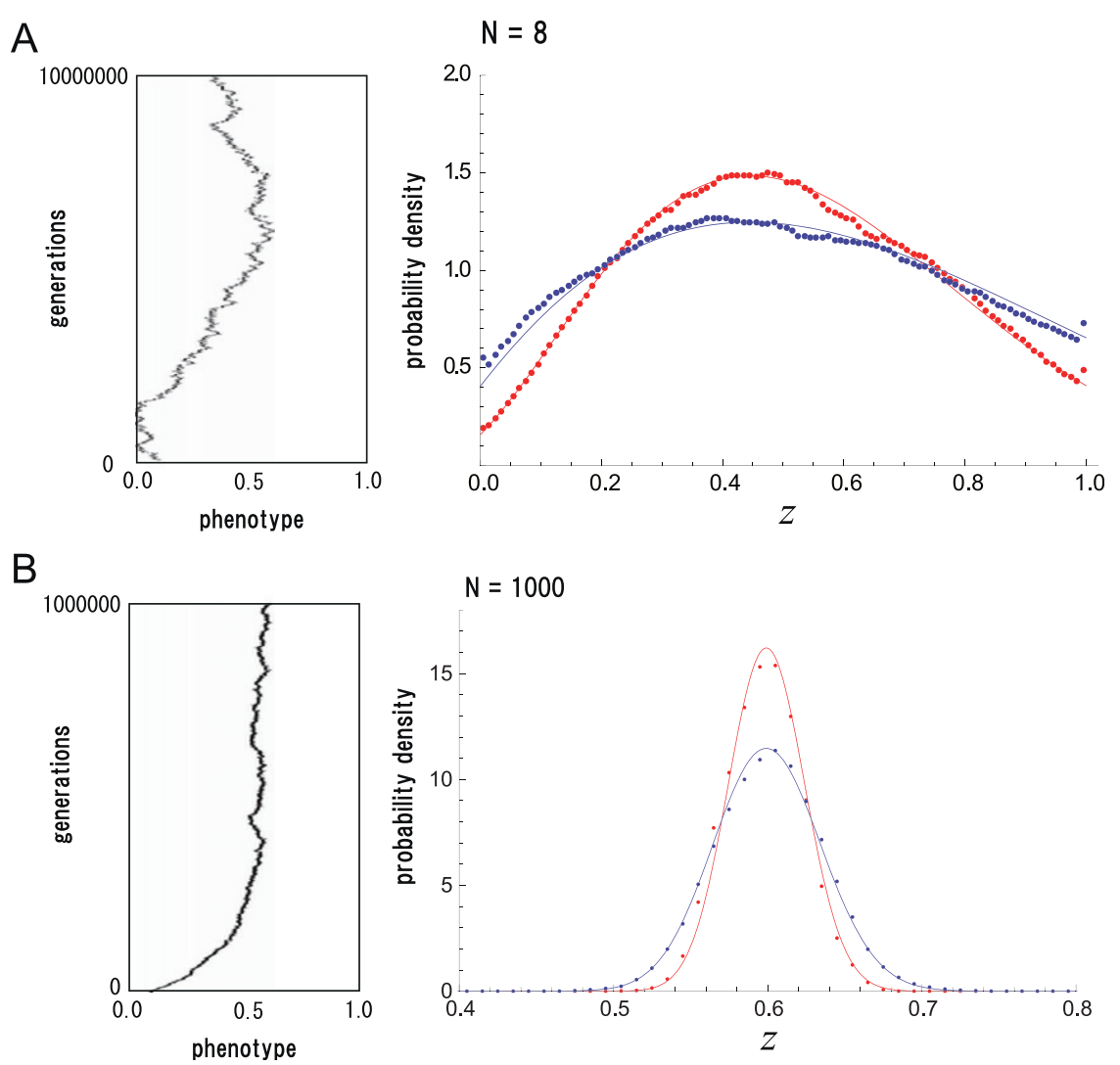

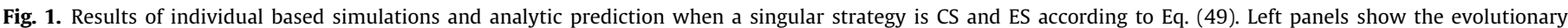

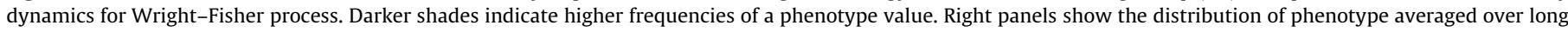

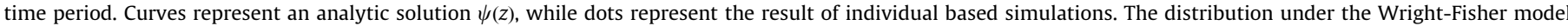

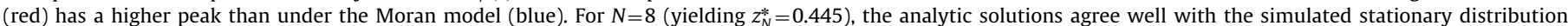

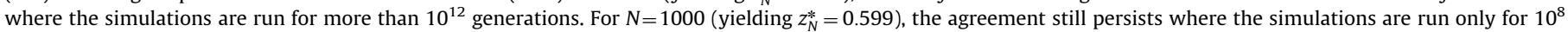

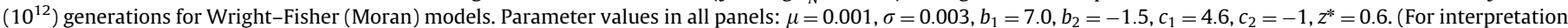
of the references to color in this figure caption, the reader is referred to the web version of this article.) 

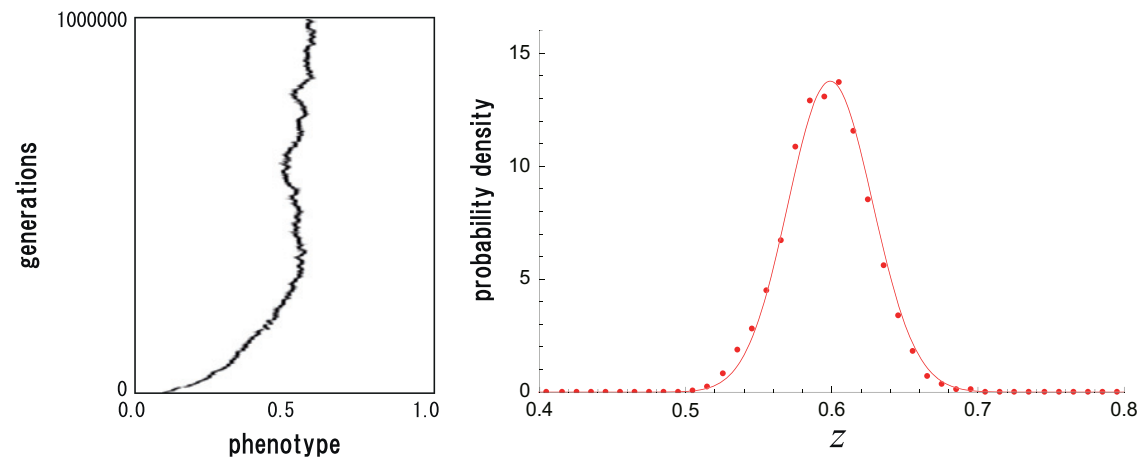

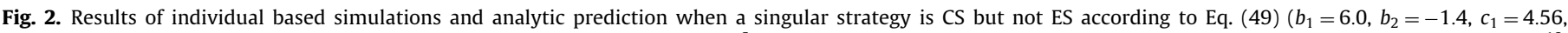

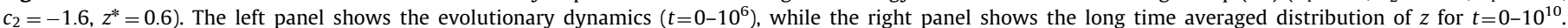

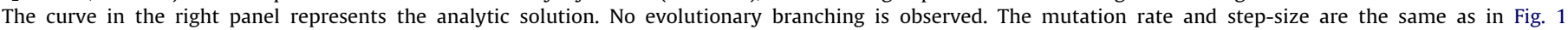
( $\mu=0.001, \sigma=0.003$ ). Wright-Fisher model is used with a population size $N=1000$.

effects as

$Q_{S}: f_{1}\left(z^{*}\right)-f_{2}\left(z^{*}\right) /(N-1)=0$,

$Q_{\mathrm{CS}}: f_{11}\left(z^{*}\right)+f_{12}\left(z^{*}\right)(N-2) /(N-1)-f_{22}\left(z^{*}\right) /(N-1)<0$,

$Q_{\mathrm{ES}}: f_{11}\left(z^{*}\right)-f_{22}\left(z^{*}\right) /(N-1)<0$.

\subsection{Game with branching}

As an example, we follow the model of Doebeli et al. (2004). Let $z$ represent the amount of investment in public goods, which defines the payoff of a player with strategy $z_{1}$ when meeting a $z_{2}$ player as

$f\left(z_{1}, z_{2}\right)=1+\left[b_{2}\left(z_{1}+z_{2}\right)^{2}+b_{1}\left(z_{1}+z_{2}\right)\right]-\left[c_{2}\left(z_{1}\right)^{2}+c_{1} z_{1}\right]$

where the public goods benefit returning to an actor depends on the sum of the amounts of investments by both players, while the cost is paid by the actor alone. Then we have

$Q_{\mathrm{s}}: z_{N}^{*}=\frac{c_{1}-\alpha_{N} b_{1}}{4 \alpha_{N} b_{2}-2 c_{2}}$,

$Q_{\mathrm{CS}}: 4 \alpha_{N} b_{2}-2 c_{2}<0$,

$Q_{\mathrm{ES}}: 2 \alpha_{N} b_{2}-2 c_{2}<0$,

where $\alpha_{N} \equiv(N-2) /(N-1)$. When population size becomes infinitely large, $\alpha_{N}$ tends to unity, and one has

$Q_{\mathrm{S}}: z^{*}=\left(c_{1}-b_{1}\right) /\left(4 b_{2}-2 c_{2}\right)$,

$Q_{\mathrm{CS}}: 4 b_{2}-2 c_{2}<0$,

$Q_{\mathrm{ES}}: 2 b_{2}-2 c_{2}<0$,

which matches the expressions of Doebeli et al. (2004). By comparing Eq. (49) to Eq. (50) one sees that in a finite population the effective benefits of cooperation produced by investment is decreased by the factor $\alpha_{N}$ relative to the infinite population case.

\subsection{Numerical-analytical comparisons}

Doebeli et al. (2004) have shown by individual based simulations that evolutionary branching occurs in a population of size $N=10000$ for appropriate mutation parameters when $z^{*}$ is CS but not ES according to Eq. (50). When population size is small, we must use Eq. (49) instead of Eq. (50) to obtain the singular strategy $z_{N}^{*}$ and the stability conditions. In order to compare our results to those obtained in simulations, where mutations are present and which leads to a long-run phenotypic stationary distribution, we evaluate the stationary distribution, $\psi(z)$, arising from the game described by Eq. (48). To that aim we use the gradient

$\dot{\pi}(z)=\frac{(N-1)}{N(1+s) f}\left[b_{1}+4 b_{2} z-c_{1}-2 c_{2} z-\frac{b_{1}+4 b_{2} z}{N-1}\right]$,

which is obtained by putting Eq. (48) into our analytic results and where $s$ is the survival probability of an individual, which is $s=0$ in the Wright-Fisher process and by setting $s=1$ one captures the Moran process (Eqs. (A.45)-(A.52) in Online Supplement).

The stationary distribution is compared in Figs. 1-2 to that obtained from individual based simulations in which the mutation process is explicitly modeled and thus more than two different phenotypes can simultaneously coexist at each snapshot in the population and phenotypic effects of mutants can be large (see Online Supplement "Individual based simulation" for details). When the mutation rate, $\mu$, and the variance of the probability distribution of mutation step size, $\sigma^{2}$, are small, the simulation results agree well with the analytic predictions when a singular strategy is both ES and CS, where the stationary distribution is centered at $z_{N}^{*}$ predicted by Eq. (49) (Fig. 1). In the case of a small population $(N=8)$, the distribution is broad due to strong genetic drift but well approximated by the stationary distribution $\psi(z)$ (Fig. 1). In the case of $N=1000$, the population is large enough to have $z_{N}^{*} \simeq z^{*}$ but not large enough to obtain a sharp peak at $z_{N}^{*}$ in the stationary distribution so that drift still plays an important role (Fig. 1), which is well predicted by the stationary distribution. Finally, the distribution becomes broader when we adopt the Moran model instead of the Wright-Fisher model, which is again analytically predicted by Eq. (44) with Eq. (51).

The agreement between simulations and analytical predictions is good not only when the singular point is both CS and ES (as in Fig. 1), but also when the singular point is CS and non-ES (i.e., evolutionary branching point) as long as the mutational effects ( $\mu$ and $\sigma^{2}$ ) are small (Fig. 2).

Next we run simulations where mutational effects and rates are no longer small (Fig. 3). When the population is small $(N=8)$, the realized stationary distribution has still the same features as that predicted from a two-allele model; the distribution has a peak at each snapshot but the position of the peak moves around (compare panel A Figs. 1-3). This result is consistent with the result of the two-allele model because it claims that population should spend more time near $z=z_{N}^{*}$ when population can have at most two different kinds of phenotypes, $z$ and $z+\delta$, at each time. As long as our two-allele approximation is good, the only stability condition is Eq. (49), which converges to the CS condition in the infinite population limit, and evolutionary branching does 

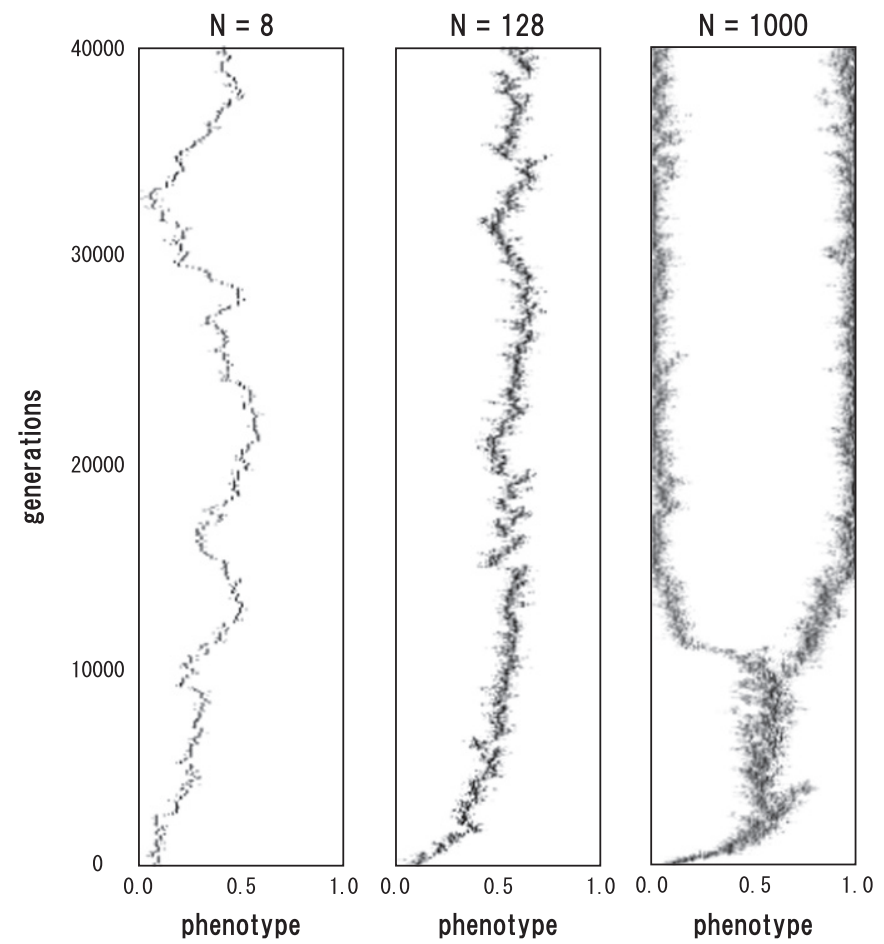

Fig. 3. Evolutionary dynamics in individual based simulations when a singular strategy is CS but not ES according to Eq. (49). The same parameter as in Fig. 2 are used except that the mutation rate and step size are increased. Singular values, $z_{N}^{*}$. are $0.364,0.591$ and 0.599 , respectively for $N=8,128$, and 1000 . Results for Wright-Fisher models are shown. Parameter values: $\mu=0.01, \sigma=0.02, b_{1}=6.0$, $b_{2}=-1.4, c_{1}=4.56, c_{2}=-1.6, z^{*}=0.6$.

not occur. When population size is intermediate $(N=128)$, the approximation is no longer good and some level of phenotypic variance persists at each snapshot. However, it is not large enough to lead to evolutionary branching. As we increase population size more $(N=1000)$, the variance is also increased and evolutionary branching occurs. In the last simulation showing branching dynamics, the average number of segregating alleles (different alleles simultaneously coexisting) is roughly 80 , which is far more than two and the outcome of evolution is now much different from that predicted from the two-allele approximation (compare Figs. 2 and 3).

\section{Discussion}

\subsection{Summary of the approach}

We have derived stability conditions for the long-term evolution of a continuous phenotype in a finite population where mutant-mutant interactions are explicitly taken into account. These conditions are based from considerations of the stationary average frequency of a mutant allele in the presence of vanishing mutation rates in a two-allele model. We focused on a two-allele system because this represents the natural extension to finite populations (stochastic system) of standard mutant-resident model of evolutionary game theory in infinite populations (e.g., Eshel, 1983; Taylor, 1989; Lessard, 1990; Geritz et al., 1998), and provides the possibility to analytically determine stability conditions. We calculate the stationary average frequency from the fixation probability, as we are interested in long-term evolution in small populations where invasion and fixation repeatedly occurs.

In order to obtain the stability conditions, we have derived perturbation expansions of the asymptotic frequencies (fixation probability and stationary average frequency) of a mutant allele with a moment closure method based on a Markov chain approach, which encapsulates many different life-cycle assumptions (e.g., reproductive scheme or underlying neutral process such as Wright-Fisher, Moran, etc.). As in standard deterministic models, we considered the first- and second-order effects of selection intensity on allele frequency change, thereby assuming weak selection in the sense that phenotypic difference between resident and mutant are small. But owing to finite population size, mutant-mutant interactions are taken into account. Thus, stability conditions may not be determined by payoff alone as in deterministic systems (e.g., Eshel, 1983; Taylor, 1989; Lessard, 1990; Geritz et al., 1998), but may depend on the features of the life-cycle assumption.

\subsection{Summary of the results}

As in previous analyses (Rousset, 2003; Lessard and Ladret, 2007; Lehmann and Rousset, 2009), we expressed the perturbation expansion of the mutant fixation probability in terms of fitness effects weighted by coalescence times (Eqs. (30) and (31)), which capture the effect of mutant-mutant interactions in a finite population. Unexpectedly, we find that the perturbation expansion of the stationary average mutant frequency can be expressed in terms of fitness effects weighted by the total branch length in number of generations of a coalescent tree (Eqs. (34) and (35)). This symmetry may be interesting to explore further.

Turning to the stability conditions, we find that the condition for a strategy to be a singular point $\left(Q_{S}\right)$, where first-order effects in selection intensity vanish, does not depend on the specificities of the life-cycle assumption. This condition can be directly obtained from the selection gradient on a mutant allele (or invasion fitness), which is proportional to the first-order effects in selection intensity on both the fixation probability or the stationary average frequency of a mutant allele (Eqs. (30) and (34)). This result has been derived previously (Rousset and Billiard, 2000; Taylor et al., 2007), and it shows that the determination of singular points for a continuous trait in a stochastic system (finite population size) is qualitatively the same as that in a deterministic system (infinite population size).

Since the condition of convergence stability $\left(Q_{\mathrm{cS}}\right)$ in finite populations is defined from first-order effects of selection, it qualitatively agree with that for infinite population size, irrespective of whether we define it based on the fixation probability $(\dot{\pi}(z))$ or the stationary average frequency of the mutant allele $(\dot{\rho}(z))$.

We find that our second-order stability condition $\left(Q_{\mathrm{TS}}\right.$, Eq. $\left.(43)\right)$, which is computed from second-order effects in selection intensity as is the standard condition of evolutionary stability, reduces to the condition of convergence stability $\left(Q_{\mathrm{TS}}=Q_{\mathrm{CS}}\right)$. Hence, under the traits substitution sequence assumption, a positive answer to the question of convergence stability [question (ii) of Eshel, 1983 described in the Introduction] guaranties a positive answer to the question of evolutionary stability [question (i) of Eshel, 1983 described in the Introduction], and vice versa.

It is well known that evolutionary branching can occur at a singular point that is convergence stable in a population of large size with sufficiently large mutation rate, and that these points are well predicted by the traditional ES condition (Geritz et al., 1997; Dieckmann and Doebeli, 1999; Doebeli et al., 2004). But whether such branching actually occurs critically rely on the number of different alleles coexisting in the population. Comparing Figs. 2 and 3 illustrates that the interaction between mutation rate and population size plays a crucial in role in the evolutionary dynamics, a feature that has been pointed out before (Waxman and Gavrilets, 2005; Barton and Polechova, 2005; Champagnat 
et al., 2006). Even if the mutation rate is larger than the bound ensuring the trait substitution sequence $[\mu<1 /(N \log (N))$, Champagnat et al., 2006; Wu et al., 2012], the simulation results do not deviate significantly from the analytical results. However, none of the $Q_{S}, Q_{E S}$, or $Q_{\mathrm{TS}}$ conditions actually include mutation rate nor mutation step size.

The curvature of the stationary phenotypic distribution function (Eq. (44)) at a singular point is indicative of its stability by convergence and it may be characterized by Hamilton's (1964) inclusive fitness effect in spatially or family structured populations (Lehmann, 2012). This is parallel but different from the result that the curvature of the invasion fitness is indicative of the ES condition (Eq. (14)). Thus, for two-allele models, long-term phenotypic evolution might be characterized by the inclusive fitness effect alone (based on first order effects in selection intensity), which applies to class-structured population of finite size (Rousset, 2004; Rousset and Ronce, 2004; Taylor et al., 2007).

In a general case with large mutational effects, two-allele models are no longer appropriate. Evolutionary branching may then occur and the traditional ES condition is usually used to determine branching. But in finite populations one cannot predict whether branching occurs without knowing more details about the mutation scheme, so checking the traditional evolutionary stability conditions is not sufficient. In order to survey the conditions under which evolutionary branching occurs, it seems necessary to better understand the effect of two additional factors: the number of alleles that can simultaneously segregate in the system and the mutation step size distribution.

\section{Acknowledgments}

We thank Yoh Iwasa, Yutaka Kobayashi, Hisashi Ohtsuki, Jorge Peña, Jeremy van Cleve and anonymous reviewers for useful comments. This work is supported by PRESTO, Japan Science and Technology Agency and by Swiss NSF Grant PP00P3-123344.

\section{Appendix A. Supplementary data}

Supplementary data associated with this article can be found in the online version at http://dx.doi.org/10.1016/j.jtbi.2012.05.023.

\section{References}

Akçay, E., Van Cleve, J., 2012. Behavioral responses in structured populations pave the way to group optimality. Am. Nat. 179, 257-269.

Barton, N.H., Polechova, J., 2005. The limitations of adaptive dynamics as a model of evolution. J. Evol. Biol. 18, 1186-1190.

Bishop, D., Cannings, C., 1978. A generalized war of attrition. J. Theor. Biol. 70, 85-124.

Bulmer, M., 1994. Theoretical Evolutionary Ecology. Sinauer Associates.

Champagnat, N., Ferrière, R., Meleard, S., 2006. Unifying evolutionary dynamics: from individual stochastic processes to macroscopic models. Theor. Popul. Biol. 69, 297-321.

Champagnat, N., Lambert, A., 2007. Evolution of discrete populations and the canonical diffusion of adaptive dynamics. Ann. Appl. Probab. 17, 102-155.

Champagnat, N., Méléard, S., 2011. Polymorphic evolution sequence and evolutionary branching. Probab. Theor. Relat. Fields 151, 45-94.

Crow, J.F., Kimura, M., 1970. An Introduction to Population Genetics Theory. Harper and Row, New York.

Dercole, F., Rinaldi, S., 2008. Analysis of Evolutionary Processes: The Adaptive Dynamics Approach and Its Applications. Princetion University Press, Princeton, NJ.

Dieckmann, U., Doebeli, M., 1999. On the origin of species by sympatric speciation. Nature 400, 354-357.

Doebeli, M., Hauert, C., Killingback, T., 2004. The evolutionary origin of cooperators and defectors. Science 306, 859-862.
Donnelly, P., Tavaré, S., 1995. Coalescents and genealogical structure under neutrality. Annu. Rev. Genet. 29, 401-421.

Eshel, I., 1983. Evolutionary and continuous stability. J. Theor. Biol. 103, 99-111.

Ewens, W.J., 2004. Mathematical Population Genetics. Springer-Verlag, New York.

Fudenberg, D., Imhof, L.A., 2006. Imitation processes with small mutations. J. Econ. Theory 131, 251-262.

Gandon, S., Rousset, F., 1999. Evolution of stepping-stone dispersal rates. Proc. R. Soc. London Ser. B Biol. Sci. 221, 2507-2513.

Geritz, S.A.H., Kisdi, E., Meszéna, G., Metz, J.A.J., 1998. Evolutionarily singular strategies and the adaptive growth and branching of the evolutionary tree. Evol. Ecol. 12, 35-57.

Geritz, S.A.H., Metz, J.A.J., Kisdi, E., Meszéna, G., 1997. Dynamics of adaptation and evolutionary branching. Phys. Rev. Lett. 78, 2024-2027.

Gillespie, J.H., 1991. The Causes of Molecular Evolution. Oxford University Press, Oxford.

Hamilton, W.D., 1964. The genetical evolution of social behaviour, I. J. Theor. Biol. 7, 1-16.

Hammerstein, P., 1996. Darwinian adaptation, population genetics and the streetcar theory of evolution. J. Math. Biol. 34, 511-532.

Kirkpatrick, M., Johnson, T., Barton, N., 2002. General models of multilocus evolution. Genetics 161, 1727-1750.

Lehmann, L., 2012. The stationary distribution of a continuously varying strategy in a class-structured population under mutation-selection-drift balance. J. Evol. Biol. 25, 770-787.

Lehmann, L., Rousset, F., 2009. Perturbation expansions of multilocus fixation probabilities for frequency-dependent selection with applications to the Hill-Robertson effect and to the joint evolution of helping and punishment. Theor. Popul. Biol. 76, 35-51.

Leimar, O., 2009. Multidimensional convergence stability. Evol. Ecol. Res. 11, 191-208.

Lessard, S., 1990. Evolutionary stability: one concept, several meanings. Theor. Popul. Biol. 37, 159-170.

Lessard, S., 2005. Long-term stability from fixation probabilities in finite populations: new perspectives for ESS theory. Theor. Popul. Biol. 68, 19-27.

Lessard, S., 2011. On the robustness of the extension of the one-third law of evolution to the multi-player game. Dyn. Games Appl. 1, 408-418.

Lessard, S., Ladret, V., 2007. Fixation probability for a beneficial allele and a mutant strategy in a linear game under weak selection in a finite island model. Theor. Popul. Biol. 72, 409-425.

Maynard-Smith, J., 1982. Evolution and the Theory of Games. Cambridge University Press, Cambridge.

Metz, J.J., Geritz, S.A.H., Meszéna, G., Jacobs, F.J.A., van Heerwaarden, J., 1996. Adaptive dynamics: a geometrical study of the consequences of nearly faithful reproduction. In: van Strien, S.J., Verduyn Lunel, S.M. (Eds.), Stochastic and Spatial Structures of Dynamical Systems. North-Holland, Amsterdam, pp. 183-231.

Nowak, M., Sasaki, A., Taylor, C., Fudenberg, D., 2004. Emergence of cooperation and evolutionary stability in finite populations. Nature $428,646-650$.

Ohtsuki, H., 2010. Stochastic evolutionary dynamics of bimatrix games. J. Theor. Biol. 264, 136-142.

Otto, S.P., Day, T., 2007. A Biologist's Guide to Mathematical Modeling in Ecology and Evolution. Princeton University Press, Princeton, NJ.

Parker, G.A., Maynard Smith, J., 1990. Optimality theory in evolutionary biology. Science 349, 27-33.

Rousset, F., 2003. A minimal derivation of convergence stability measures. J. Theor. Biol. 221, 665-668.

Rousset, F., 2004. Genetic Structure and Selection in Subdivided Populations. Princeton University Press, Princeton, NJ.

Rousset, F., Billiard, S., 2000. A theoretical basis for measures of kin selection in subdivided populations: finite populations and localized dispersal. J. Evol. Biol. 13, 814-825.

Rousset, F., Ronce, O., 2004. Inclusive fitness for traits affecting metapopulation demography. Theor. Popul. Biol. 65, 127-141.

Tarnita, C., Antal, T., Nowak, M., 2009. Mutation-selection equilibrium in games with mixed strategies. J. Theor. Biol. 261, 50-57.

Taylor, P.D., 1989. Evolutionary stability in one-parameter models under weak selection. Theor. Popul. Biol. 36, 125-143.

Taylor, P.D., 1994. Sex ratio in a stepping-stone population with sex-specific dispersal. Theor. Popul. Biol. 45, 203-218.

Taylor, P.D., Day, T., Wild, G., 2007. From inclusive fitness to fixation probability in homogeneous structured populations. J. Theor. Biol. 249, 101-110.

Vincent, T.L., Brown, J.S., 2005. Evolutionary Game Theory, Natural Selection, and Darwinian Dynamics. Cambridge University Press, Cambridge.

Waxman, D., Gavrilets, S., 2005. 20 questions on adaptive dynamics. J. Evol. Biol. 18, 1139-1154.

Weibull, J.W., 1997. Evolutionary Game Theory. MIT Press, MA.

Wild, G., Taylor, P., 2004. Fitness and evolutionary stability in game theoric models of finite populations. Proc. R. Soc. London Ser. B Biol. Sci. 271, 2345-2349.

Wright, S., 1931. Evolution in Mendelian populations. Genetics 16, 97-159.

Wu, B., Gokhale, C.S., Wang, L., Traulsen, A., 2012. How small are small mutation rates? J. Math. Biol. 64, 803-827. 


\section{Online Supplement}

"Evolutionary and convergence stability for continuous phenotypes in finite populations derived from two-allele models" by Joe Yuichiro Wakano and Laurent Lehmann

\section{Definitions of $f_{i}$ and $f_{i j}$}

Considering that a pairwise payoff function $f\left(z_{1}, z_{2}\right)$ is a smooth function, we have

$$
f\left(z+\delta_{1}, z+\delta_{2}\right)=f+f_{1} \delta_{1}+f_{2} \delta_{2}+f_{11} \frac{\delta_{1}^{2}}{2}+f_{12} \delta_{1} \delta_{2}+f_{22} \frac{\delta_{2}^{2}}{2}+\ldots,
$$

where

$$
\begin{aligned}
& f \equiv f(z, z) \\
& \left.f_{1} \equiv \frac{\partial f\left(z_{1}, z_{2}\right)}{\partial z_{1}}\right|_{z_{1}=z_{2}=z} \\
& \left.f_{2} \equiv \frac{\partial f\left(z_{1}, z_{2}\right)}{\partial z_{2}}\right|_{z_{1}=z_{2}=z} \\
& \left.f_{11} \equiv \frac{\partial^{2} f\left(z_{1}, z_{2}\right)}{\partial z_{1}^{2}}\right|_{z_{1}=z_{2}=z} \\
& \left.f_{22} \equiv \frac{\partial^{2} f\left(z_{1}, z_{2}\right)}{\partial z_{2}^{2}}\right|_{z_{1}=z_{2}=z}=\left.\frac{\partial^{2} f\left(z_{1}, z_{2}\right)}{\partial z_{2} \partial z_{1}}\right|_{z_{1}=z_{2}=z} \equiv f_{21}, \\
& \left.f_{12} \equiv \frac{\partial^{2} f\left(z_{1}, z_{2}\right)}{\partial z_{1} \partial z_{2}}\right|_{z_{1}=z_{2}=z}
\end{aligned}
$$

so that all functions are evaluated at the resident value $z$.

\section{Coefficients of the A matrix}

Here, we evaluate the remaining coefficients appearing in the recursion defined by eqs. 20-21 and we will do this for both Wright-Fisher, Moran, and Cannings process (Ewens, 2004). From eqs. 20-22, the recursion for the conditional second moment is

$$
\begin{aligned}
\mathrm{E}\left[p_{\mathrm{A} / \mathrm{A}}(t+1) \mid \mathbf{p}(t)=\mathbf{p} ; \delta\right] & =a_{21}^{\circ} p_{\mathrm{A}}+\left(1-a_{21}^{\circ}\right) p_{\mathrm{A} / \mathrm{A}} \\
+ & \delta\left[\dot{a}_{21} p_{\mathrm{A}}+\dot{a}_{22} p_{\mathrm{A} / \mathrm{A}}-\left(\dot{a}_{21}+\dot{a}_{22}\right) p_{\mathrm{A} / \mathrm{A} / \mathrm{A}}\right]+O\left(\delta^{2}\right)
\end{aligned}
$$

where $p_{\mathrm{A}}, p_{\mathrm{A} / \mathrm{A}}$, and $p_{\mathrm{A} / \mathrm{A} / \mathrm{A}}$ are calculated from the realization of the allele frequency distribution $\mathbf{p}$ in the parental generation. In order to evaluate the coefficients appearing in 
this second order approximation, we first note that regardless of the intensity of selection we have from eq. 15 that

$$
\mathrm{E}\left[p_{\mathrm{A} / \mathrm{A}}(t+1) \mid \mathbf{p}(t)=\mathbf{p} ; \delta\right]=\frac{1}{N(N-1)} \sum_{i} \sum_{j \neq i} \operatorname{Pr}\left(p_{i}(t+1)=p_{j}(t+1)=1 \mid \mathbf{p}(t)=\mathbf{p} ; \delta\right),
$$

where $\operatorname{Pr}\left(p_{i}(t+1)=p_{j}(t+1)=1 \mid \mathbf{p}(t)=\mathbf{p} ; \delta\right)$ is the conditional probability that both individual $i$ and $j$ carry the mutant allele in the descendant generation given realization $\mathbf{p}$. We will first obtain an expression for $\operatorname{Pr}\left(p_{i}(t+1)=p_{j}(t+1)=1 \mid \mathbf{p}(t)=\mathbf{p} ; \delta\right)$, and then calculate the coefficient in eq. A-4 from it.

\section{Wright-Fisher process}

For the Wright-Fisher process, the probability $\operatorname{Pr}\left(p_{i}(t+1)=1 \mid \mathbf{p}(t)=\mathbf{p} ; \delta\right)$ that individual $i$ carries the mutant allele in the descendant generation conditional on realization $\mathbf{p}$ in the parental generation is

$$
\operatorname{Pr}\left(p_{i}(t+1)=1 \mid \mathbf{p}(t)=\mathbf{p} ; \delta\right)=\frac{1}{N} \sum_{k=1}^{N} w_{k}(\mathbf{z}(\mathbf{p})) p_{k} .
$$

Since the random variables $p_{i}(t+1)$ and $p_{j}(t+1)$ are independent for $i \neq j$, i.e., the sampling of the parent of individual $i$ and that of $j$ are independent and we have

$$
\operatorname{Pr}\left(p_{i}(t+1)=p_{j}(t+1)=1 \mid \mathbf{p}(t)=\mathbf{p} ; \delta\right)=\frac{1}{N^{2}} \sum_{k=1}^{N} \sum_{l=l}^{N} w_{k}(\mathbf{z}(\mathbf{p})) w_{l}(\mathbf{z}(\mathbf{p})) p_{k} p_{l} .
$$

Substituting this expression into eq. A-4 and applying a first order Taylor expansion of the fitness function around $\delta=0$ yields

$$
\mathrm{E}\left[p_{\mathrm{A} / \mathrm{A}}(t+1) \mid \mathbf{p}(t)=\mathbf{p} ; \delta\right]=\frac{1}{N^{2}} \sum_{k=1}^{N} \sum_{l=l}^{N} p_{k} p_{l}\left[1+\delta\left(\sum_{m=1}^{N} w_{k, m} p_{m}+\sum_{n=l}^{N} w_{l, n} p_{n}\right)\right]+O\left(\delta^{2}\right) .
$$

The right hand side equals

$$
\frac{1}{N^{2}}\left[N p_{\mathrm{A}}+N(N-1) p_{\mathrm{A} / \mathrm{A}}\right]+\frac{2 \delta}{N^{2}} X+O\left(\delta^{2}\right)
$$

where

$$
X \equiv \sum_{k=1}^{N} \sum_{l=l}^{N} w_{k, k} p_{k} p_{l}+\sum_{k=1}^{N} \sum_{l=l}^{N} \sum_{m \neq k}^{N} w_{k, m} p_{k} p_{l} p_{m}
$$


Using the symmetry (eq. 11), we have

$$
X=w_{k, k}\left(\sum_{k=1}^{N} p_{k}+\sum_{k=1}^{N} \sum_{l \neq k}^{N} p_{k} p_{l}\right)+w_{k, m}\left(2 \sum_{k=1}^{N} \sum_{m \neq k}^{N} p_{k} p_{m}+\sum_{k=1}^{N} \sum_{m \neq k}^{N} \sum_{l \neq k, m}^{N} p_{k} p_{l} p_{m}\right)
$$

and using eq. 15 gives

$$
\begin{aligned}
X=w_{k, k}\left[N p_{\mathrm{A}}+N(N-1) p_{\mathrm{A} / \mathrm{A}}\right] & \\
& +w_{k, m}\left[2 N(N-1) p_{\mathrm{A} / \mathrm{A}}+N(N-1)(N-2) p_{\mathrm{A} / \mathrm{A} / \mathrm{A}}\right] .
\end{aligned}
$$

We now substitute $w_{k, m}=-w_{k, k} /(N-1)$ into this equation (obtained from the zero sum property of the partial derivatives (Rousset, 2004)), which gives

$$
X=w_{k, k} N\left[p_{\mathrm{A}}+(N-3) p_{\mathrm{A} / \mathrm{A}}-(N-2) p_{\mathrm{A} / \mathrm{A} / \mathrm{A}}\right] .
$$

Combining these results, we finally obtain

$$
\begin{aligned}
& \mathrm{E}\left[p_{\mathrm{A} / \mathrm{A}}(t+1) \mid \mathbf{p}(t)=\mathbf{p} ; \delta\right]=\frac{1}{N} p_{\mathrm{A}}+\frac{N-1}{N} p_{\mathrm{A} / \mathrm{A}} \\
& +\delta w_{i, i}\left[\frac{2}{N} p_{\mathrm{A}}+\frac{2(N-3)}{N} p_{\mathrm{A} / \mathrm{A}}-\frac{2(N-2)}{N} p_{\mathrm{A} / \mathrm{A} / \mathrm{A}}\right]+O\left(\delta^{2}\right)
\end{aligned}
$$

which shows that

$$
\begin{aligned}
a_{21}^{\circ} & =\frac{1}{N} \\
a_{22}^{\circ} & =\frac{N-1}{N} \\
\dot{a}_{21} & =\frac{2}{N} w_{i, i} \\
\dot{a}_{22} & =\frac{2(N-3)}{N} w_{i, i} . \\
a_{23}^{\circ} & =-\frac{2(N-2)}{N} w_{i, i} .
\end{aligned}
$$

In order to close the system (eqs. 20-21), we need the recursion for $\rho_{\mathrm{A} / \mathrm{A} / \mathrm{A}}(t)$ under neutrality. By a similar calculation, we obtain

$$
\mathrm{E}\left[p_{\mathrm{A} / \mathrm{A} / \mathrm{A}}(t+1) \mid \mathbf{p}(t)=\mathbf{p} ; \delta\right]=\frac{1}{N^{2}} p_{\mathrm{A}}+\frac{3(N-1)}{N^{2}} p_{\mathrm{A} / \mathrm{A}}+\frac{(N-1)(N-2)}{N^{2}} p_{\mathrm{A} / \mathrm{A} / \mathrm{A}}+O(\delta),
$$

which shows that $a_{31}^{\circ}=1 / N^{2}$ and $a_{32}^{\circ}=3(N-1) / N^{2}$. 


\section{Moran process}

For the Moran process, we assume that the $N$ haploid parents produce a very large number of haploid offspring and that exactly one randomly sampled parent dies after reproduction, which is then replaced by a juvenile randomly sampled from the pool of offspring. We also assume that the behavior of individuals affect fecundity. With this we can write the fitness of individual $i$ as

$$
w_{i}(\mathbf{z}(\mathbf{p}))=\frac{N-1}{N}+\frac{1}{N} \tilde{w}_{i}(\mathbf{z}(\mathbf{p})),
$$

where

$$
\tilde{w}_{k} \equiv \frac{F_{k}(\mathbf{z})}{\bar{F}(\mathbf{z})}
$$

is the fecundity of individual $k$ relative to the average fecundity. Since this fitness function determines the dynamics of the mean allele frequency (eq. 9), we have from eqs. 10-16 that

$$
\begin{aligned}
\dot{a}_{11} & =\tilde{w}_{i, i} / N \\
\ddot{a}_{11} & =\tilde{w}_{i, i i} / N \\
\ddot{a}_{12} & =\left[2(N-1) \tilde{w}_{i, i j}+(N-1) \tilde{w}_{i, j j}\right] / N .
\end{aligned}
$$

In the Moran process, the random variables $p_{i}(t+1)$ and $p_{j}(t+1)$ are not independent because an individual $i$ cannot be a newborn if an individual $j$ is a newborn, and the conditional probability that both individual $i$ and $j$ carry the mutant allele in the descendant generation is given by

$$
\begin{aligned}
& \operatorname{Pr}\left.p_{i}(t+1)=p_{j}(t+1)=1 \mid \mathbf{p}(t)=\mathbf{p} ; \delta\right) \\
& \quad=\left(\frac{N-2}{N}\right) p_{i} p_{j}+\frac{1}{N} \times\left(\frac{1}{N} \sum_{k=1}^{N} \tilde{w}_{k}(\mathbf{z}(\mathbf{p})) p_{k} p_{i}+\frac{1}{N} \sum_{k=1}^{N} \tilde{w}_{k}(\mathbf{z}(\mathbf{p})) p_{k} p_{j}\right),
\end{aligned}
$$

where $(N-2) / N$ is the probability that individual $i$ and $j$ have survived from the parental generation, while with probability $1 / N$ either individual has died and has been replaced by an offspring. In each of these two events, the new offspring descends from individual $k$ with probability $\tilde{w}_{k} / N$, where $\tilde{w}_{k} \equiv F_{k} / \bar{F}$ is the fecundity of individual $k$ relative to the average fecundity. Substituting the conditional probability into eq. A-4 yields

$$
\mathrm{E}\left[p_{\mathrm{A} / \mathrm{A}}(t+1) \mid \mathbf{p}(t)=\mathbf{p} ; \delta\right]=\frac{N-2}{N} p_{\mathrm{A} / \mathrm{A}}+\frac{2}{N} \times\left(\frac{1}{N^{2}} \sum_{k=1}^{N} \sum_{j=1}^{N} \tilde{w}_{k} p_{k} p_{j}\right)
$$


We have the following Taylor expansion

$$
\frac{1}{N^{2}} \sum_{k=1}^{N} \sum_{j=1}^{N} \tilde{w}_{k} p_{k} p_{j}=\sum_{k=1}^{N} \sum_{j=1}^{N}\left(1+\delta \sum_{i=1}^{N} \tilde{w}_{k, i} p_{i}\right) p_{k} p_{j}+O\left(\delta^{2}\right)
$$

which yields

$$
\frac{1}{N^{2}} \sum_{k=1}^{N} \sum_{j=1}^{N} \tilde{w}_{k} p_{k} p_{j}=\frac{1}{N^{2}}\left[N p_{\mathrm{A}}+N(N-1) p_{\mathrm{A} / \mathrm{A}}\right]+\frac{\delta}{N^{2}} Y+O\left(\delta^{2}\right),
$$

where

$$
Y \equiv \sum_{k=1}^{N} \sum_{j=1}^{N} \sum_{i=1}^{N} \tilde{w}_{k, i} p_{i} p_{k} p_{j},
$$

which has the same structure as eq. A-9. Using eq. 11 and the zero sum property of the partial derivatives (Rousset, 2004), we thus get

$$
Y=\tilde{w}_{k, k} N\left[p_{\mathrm{A}}+(N-3) p_{\mathrm{A} / \mathrm{A}}-(N-2) p_{\mathrm{A} / \mathrm{A} / \mathrm{A}}\right]
$$

and with this, we finally obtain

$$
\begin{aligned}
& \mathrm{E}\left[p_{\mathrm{A} / \mathrm{A}}(t+1) \mid \mathbf{p}(t)=\mathbf{p} ; \delta\right]=\frac{2}{N^{2}} p_{\mathrm{A}}+\frac{N^{2}-2}{N^{2}} p_{\mathrm{A} / \mathrm{A}} \\
& +\delta\left[\frac{2}{N^{2}} p_{\mathrm{A}}+\frac{2(N-3)}{N^{2}} p_{\mathrm{A} / \mathrm{A}}-\frac{2(N-2)}{N^{2}} p_{\mathrm{A} / \mathrm{A} / \mathrm{A}}\right] \tilde{w}_{k, k}+O\left(\delta^{2}\right)
\end{aligned}
$$

which gives

$$
\begin{aligned}
a_{21}^{\circ} & =\frac{2}{N^{2}} \\
a_{22}^{\circ} & =\frac{N^{2}-2}{N^{2}} \\
\dot{a}_{21} & =\frac{2}{N^{2}} \tilde{w}_{i, i} \\
\dot{a}_{22} & =\frac{2(N-3)}{N^{2}} \tilde{w}_{i, i} . \\
\dot{a}_{23} & =-\frac{2(N-2)}{N^{2}} \tilde{w}_{i, i} .
\end{aligned}
$$

Comparing eq. 17 and eq. A-14 for the Wright-Fisher process to eq. A-18 and eq. A-26 for the Moran process shows that the neutral process under the Wright-Fisher and Moran processes involves different zeroth order coefficients but that the the first order coefficients take the same form when expressed in terms of fitness effects since $\tilde{w}_{i, i}=N w_{i, i}$, and $\tilde{w}_{i, j k}=N w_{i, i j}$. 
But importantly, since for fecundity effects are $w_{i}=F_{i} / \bar{F}$ for the Wright-Fisher process, while $\tilde{w}_{i}=F_{i} / \bar{F}$ for the Moran process, the selection coefficients in the two processes are equivalent up to a constant of proportionality $1 / N$.

In order to close the system for the Moran process, we need the recursion for $\rho_{\mathrm{A} / \mathrm{A} / \mathrm{A}}(t)$ under neutrality. Applying standard arguments (Ewens, 2004) shows that

$$
\mathrm{E}\left[p_{\mathrm{A} / \mathrm{A} / \mathrm{A}}(t+1) \mid \mathbf{p}(t)=\mathbf{p} ; \delta\right]=\frac{N-3}{N} p_{\mathrm{A} / \mathrm{A} / \mathrm{A}}+\frac{3}{N}\left(\frac{2}{N} p_{\mathrm{A} / \mathrm{A}}+\frac{N-2}{N} p_{\mathrm{A} / \mathrm{A} / \mathrm{A}}\right)+O(\delta),
$$

which shows $a_{31}^{\circ}=0$ and $a_{32}^{\circ}=6 / N^{2}$.

\section{Cannings process}

For the Cannings process, we consider that each individual adult dies with probability $s$ and is replaced by an individual randomly sampled from the pool of offspring. With behavior affecting fecundity, the fitness of individual $i$ in the Cannings process is

$$
w_{i}(\mathbf{z}(\mathbf{p}))=s+(1-s) \tilde{w}_{i}(\mathbf{z}(\mathbf{p}))
$$

and

$$
\begin{aligned}
& \operatorname{Pr}\left(p_{i}(t+1)=p_{j}(t+1)\right.=1 \mid \mathbf{p}(t)=\mathbf{p} ; \delta)=s^{2} p_{i} p_{j} \\
&+s(1-s) \times\left(\frac{1}{N} \sum_{k=1}^{N} \tilde{w}_{k}(\mathbf{z}(\mathbf{p})) p_{k} p_{i}+\frac{1}{N} \sum_{k=1}^{N} \tilde{w}_{k}(\mathbf{z}(\mathbf{p})) p_{k} p_{j}\right) \\
&+(1-s)^{2} \times\left(\frac{1}{N^{2}} \sum_{k=1}^{N} \sum_{l=l}^{N} \tilde{w}_{k}(\mathbf{z}(\mathbf{p})) \tilde{w}_{l}(\mathbf{z}(\mathbf{p})) p_{k} p_{l}\right),
\end{aligned}
$$

Substituting this equation into eq. A-4, after some calculation we have

$$
\begin{aligned}
& \mathrm{E}\left[p_{\mathrm{A} / \mathrm{A}}(t+1) \mid \mathbf{p}(t)=\mathbf{p} ; \delta\right]=s^{2} p_{\mathrm{A} / \mathrm{A}}+\left(1-s^{2}\right)\left(\frac{1}{N} p_{\mathrm{A}}+\frac{N-1}{N} p_{\mathrm{A} / \mathrm{A}}\right) \\
& +\delta w_{i, i}(1-s)\left[\frac{2}{N} p_{\mathrm{A}}+\frac{2(N-3)}{N} p_{\mathrm{A} / \mathrm{A}}-\frac{2(N-2)}{N} p_{\mathrm{A} / \mathrm{A} / \mathrm{A}}\right]+O\left(\delta^{2}\right) .
\end{aligned}
$$


In order to close the system, we need the recursion for the triplets under neutrality, which is given by

$$
\begin{aligned}
& \mathrm{E}\left[p_{\mathrm{A} / \mathrm{A} / \mathrm{A}}(t+1) \mid \mathbf{p}(t)=\mathbf{p} ; \delta\right]=s^{3} p_{\mathrm{A} / \mathrm{A} / \mathrm{A}}+3 s^{2}(1-s)\left(\frac{2}{N} p_{\mathrm{A} / \mathrm{A}}+\frac{N-2}{N} p_{\mathrm{A} / \mathrm{A} / \mathrm{A}}\right) \\
& \left\{3 s(1-s)^{2}+(1-s)^{3}\right\} \times\left(\frac{1}{N^{2}} p_{\mathrm{A}}+\frac{3(N-1)}{N^{2}} p_{\mathrm{A} / \mathrm{A}}+\frac{(N-1)(N-2)}{N^{2}} p_{\mathrm{A} / \mathrm{A} / \mathrm{A}}\right)+O(\delta),
\end{aligned}
$$

All these equations show that

$$
\begin{aligned}
& a_{21}^{\circ}=\frac{\left(1-s^{2}\right)}{N} \\
& a_{31}^{\circ}=\frac{(1-s)^{3}}{N^{2}}+\frac{3 s(1-s)^{2}}{N^{2}} \\
& a_{32}^{\circ}=\frac{3\left(1-\frac{1}{N}\right)(1-s)^{3}}{N}+\frac{6 s^{2}(1-s)}{N}+\frac{9\left(1-\frac{1}{N}\right) s(1-s)^{2}}{N} \\
& \dot{a}_{11}=(1-s) \tilde{w}_{i, i} \\
& \dot{a}_{21}=\frac{2(1-s)}{N} \tilde{w}_{i, i} \\
& \dot{a}_{22}=\frac{2(N-3)(1-s)}{N} \tilde{w}_{i, i} . \\
& \dot{a}_{23}=-\frac{2(N-2)(1-s)}{N} \tilde{w}_{i, i} .
\end{aligned}
$$

\section{Asymptotic frequencies}

\section{Method}

Here, we present the explicit expression for the fixation probability obtained from eqs. 21-25. In order to obtain this expression, we note that $\mathbf{A}(\delta)$ has a dominant unit eigenvalue and that all remaining eigenvalues of $\mathbf{A}(\delta)$ are all $<1$ (in modulus) in some small neighborhood of $\delta=0$ because all non-unit eigenvalues of $\mathbf{A}^{\circ}$ are all $<1$ (in modulus). Thus, $\lim _{t \rightarrow \infty} \mathbf{A}(\delta)^{t} \rho_{0}$ can be expressed in terms of the outer product of the right and left eigenvector associated with the unit eigenvalue of $\mathbf{A}(\delta)$ (e.g., Karlin and Taylor, 1981; Ewens, 2004). Here we only show the results of the calculation, since the derivations are complicated but straightforward application of linear algebra.

In order to interpret the resulting algebraic expressions obtained for the fixation probability, it is further useful to observe that the transition matrix $\mathbf{A}^{\circ}$ for the neutral process not only describes the forward dynamics of the moments of allelic states but since element 
$a_{i j}^{\circ}$ gives the probability that a set of genes sampled in $i$ different individuals descend from $j$ different individuals in the previous generation, $\mathbf{A}^{\circ}$ also describes a backward process: $\mathbf{u}(h+1)=\mathbf{u}(h) \mathbf{A}^{\circ}$, where $h$ proceeds from the present, $h=0$, backwards in time, and where element $u_{i}(h)$ of $\mathbf{u}(h)$ gives the probability that the ancestral lineages of a set of genes sampled from a given set of positions in the present will be in $i$ different individuals at time $h$ (Lehmann and Rousset, 2009). Standard results of Markov chain theory then show that the mean number of time steps $T_{i j}$ (sojourn time) that $i$ gene lineages spend in $j$ different individuals before coalescing into the same individual can be expressed in terms of the elements of the transition matrix $\mathbf{A}^{\circ}$, so that the perturbations of the fixation probabilities can be expressed in terms of these sojourn times.

\section{Fixation probability}

Calculating explicitly $\lim _{t \rightarrow \infty} \mathbf{A}(\delta)^{t} \rho_{0}$ in terms of the outer product of the right and left eigenvector associated with the unit eigenvalue of $\mathbf{A}(\delta)$, and rearranging the result in terms of sojourn times, the fixation probability of the mutant allele in a population consisting initially of $N_{\mathrm{A}}$ is given by

$$
\begin{aligned}
& \pi_{\mathrm{A}}\left(N_{\mathrm{A}}\right)=\frac{N_{\mathrm{A}}}{N}+\frac{N_{\mathrm{A}}\left(N-N_{\mathrm{A}}\right) T_{22}}{N(N-1)}\left[\delta \dot{a}_{11}-\delta^{2} \dot{a}_{11}\left(\dot{a}_{21}+\dot{a}_{22}\right)\left(T_{32}+T_{33}+\frac{N_{\mathrm{A}}-1}{N-2} T_{33}\right)\right. \\
& \left.+\delta^{2} \dot{a}_{11}\left(\dot{a}_{11}+\dot{a}_{22}\right) T_{22}+\frac{\delta^{2}}{2}\left\{\left(\ddot{a}_{11}+\ddot{a}_{12}\right)\left(\frac{T_{32}+T_{33}}{T_{22}}+\frac{N_{\mathrm{A}}-1}{N-2} \frac{T_{33}}{T_{22}}\right)-\ddot{a}_{12}\right\}\right]+O\left(\delta^{3}\right),
\end{aligned}
$$

where

$$
\begin{aligned}
T_{22} & =\frac{1}{a_{21}^{\circ}} \\
T_{32} & =\frac{a_{32}^{\circ}}{a_{21}^{\circ}\left(a_{31}^{\circ}+a_{32}^{\circ}\right)} \\
T_{33} & =\frac{a_{21}^{\circ}}{a_{21}^{\circ}\left(a_{31}^{\circ}+a_{32}^{\circ}\right)} .
\end{aligned}
$$

The fixation probability can be further simplified in the presence of a single initial mutant to give

$$
\begin{aligned}
\pi_{\mathrm{A}}(1)=\frac{1}{N}+\frac{T_{2}}{N}\left(\delta \dot{a}_{11}-\delta^{2} \dot{a}_{11}\right. & {\left[\left(\dot{a}_{21}+\dot{a}_{22}\right) T_{3}-\left(\dot{a}_{11}+\dot{a}_{22}\right) T_{2}\right] } \\
+ & \left.\frac{\delta^{2}}{2}\left[\frac{T_{3}}{T_{2}} \ddot{a}_{11}+\left(\frac{T_{3}-T_{2}}{T_{2}}\right) \ddot{a}_{12}\right]\right)+O\left(\delta^{3}\right),
\end{aligned}
$$


where $T_{2} \equiv T_{22}$ and $T_{3} \equiv T_{32}+T_{33}$, so that $T_{i}$ is the coalescence time of $i$ gene lineage into a single common ancestor.

Finally, we note that to the first order of selection $\pi_{\mathrm{A}}(1)>1 / N$ implies that a single resident in a population of mutants has a lower than neutral fixation probability. This can be seen from eq. A-33 since to the first order in selection intensity, we have

$$
\begin{aligned}
\pi_{\mathrm{A}}(1) & =\frac{1}{N}+\delta \frac{T_{2} \dot{a}_{11}}{N} \\
1-\pi_{\mathrm{A}}(N-1) & =\frac{1}{N}-\delta \frac{T_{2} \dot{a}_{11}}{N},
\end{aligned}
$$

and so

$$
\dot{a}_{11}>0 \quad \Leftrightarrow \quad \pi_{\mathrm{A}}(1)>\frac{1}{N} \quad \Leftrightarrow \quad 1-\pi_{\mathrm{A}}(N-1)<\frac{1}{N}
$$

holds for $\delta>0$. By contrast, at a singular point, this is no longer true since in that case the fixation probability of a single mutant and a single resident is, respectively

$$
\begin{aligned}
\pi_{\mathrm{A}}(1) & =\frac{1}{N}+\frac{\delta^{2}}{2}\left[-\ddot{a}_{12}+\left(\ddot{a}_{11}+\ddot{a}_{12}\right) \frac{T_{32}+T_{33}}{T_{22}}\right] \\
1-\pi_{\mathrm{A}}(N-1) & =\frac{1}{N}-\frac{\delta^{2}}{2}\left[-\ddot{a}_{12}+\left(\ddot{a}_{11}+\ddot{a}_{12}\right) \frac{T_{32}+2 T_{33}}{T_{22}}\right] .
\end{aligned}
$$

\section{Stationary average frequency with vanishing mutation rates}

Substituting eqs. A-33 into eq. 8, we find that the asymptotic mutant frequency in the presence of vanishing mutation rate can be written as

$$
\begin{aligned}
\rho_{\mathrm{A}}=\frac{1}{2}+\frac{L_{2}}{4}\left(\delta \dot{a}_{11}-\frac{\delta^{2}}{2} \dot{a}_{11}[\right. & \left.\left(\dot{a}_{21}+\dot{a}_{22}\right) L_{3}-\left(\dot{a}_{11}+\dot{a}_{22}\right) L_{2}\right] \\
+ & \left.\frac{\delta^{2}}{2}\left[\frac{L_{3}}{L_{2}} \ddot{a}_{11}+\left(\frac{L_{3}-L_{2}}{L_{2}}\right) \ddot{a}_{12}\right]\right)+O\left(\delta^{3}\right),
\end{aligned}
$$

where

$$
\begin{aligned}
& L_{2}=2 T_{2} \\
& L_{3}=2 T_{32}+3 T_{33}
\end{aligned}
$$

so that $L_{j}$ can be interpreted as the total branch length in number of generations of a coalescent tree involving $i$ genes. 
The stationary average frequency depends on the variable $g_{2}=L_{3} / L_{2}$, which using eq. A-40 and eq. A-34 is given by

$$
g_{2}=\frac{3 a_{21}^{\circ}+2 a_{32}^{\circ}}{2 a_{31}^{\circ}+2 a_{32}^{\circ}}
$$

The coefficient $a_{21}^{\circ}$ is the probability that two individuals $i$ and $j$ coalesce into a common ancestor (parent) in a single backward step. This probability is identical to

$$
a_{21}^{\circ}=a_{31}^{\circ}+\frac{a_{32}^{\circ}}{3}
$$

since $a_{31}^{\circ}$ is the probability that three individuals $i, j$ and $k$ coalesce into a common parent and $a_{32}^{\circ} / 3$ is the probability that two specified individuals $i$ and $j$ coalesce into a common parent and an individual $k$ does not coalesce into the common parent of $i$ and $j$. Using this relationship, we have $g_{2}=3 / 2$, which holds whenever matrix $\mathbf{A}^{\circ}$ (eq. 22) describes the dynamics of moments of allele frequencies.

\section{Explicit coefficients for fecundity effects}

Here, we present the expressions for the selection coefficients in the presence of pairwise interactions for the Wright-Fisher process. Substituting eqs. 45-46 into eq. 28 yields:

$$
\begin{aligned}
\dot{a}_{11} & =\frac{(N-1)}{N f}\left[f_{1}-\frac{f_{2}}{N-1}\right] \\
\dot{a}_{21} & =\frac{2(N-1)}{N^{2} f}\left[f_{1}-\frac{f_{2}}{N-1}\right] \\
\dot{a}_{22} & =\frac{2(N-1)(N-3)}{N^{2}}\left[f_{1}-\frac{f_{2}}{N-1}\right] \\
\ddot{a}_{11} & =-\frac{(N-1)}{N f}\left[\frac{2\left(f_{1}+f_{2}\right)}{N f}\left(f_{1}-\frac{f_{2}}{N-1}\right)-f_{11}+\frac{f_{22}}{N-1}\right] \\
\ddot{a}_{12} & =-\frac{(N-1)}{N f}\left[\frac{2(N-3)}{N f}\left(f_{1}^{2}+\frac{N-2}{N-1} f_{1} f_{2}-\frac{f_{2}^{2}}{N-1}\right)+f_{11}-2 \frac{N-2}{N-1} f_{12}-\frac{f_{22}}{N-1}\right] .
\end{aligned}
$$


At a singular point $f_{1}=f_{2} /(N-1)$, whereby

$$
\begin{aligned}
& \dot{a}_{11}=0 \\
& \dot{a}_{21}=0 \\
& \dot{a}_{22}=0 \\
& \ddot{a}_{11}=\frac{(N-1)}{N f}\left[f_{11}-\frac{f_{22}}{N-1}\right] \\
& \ddot{a}_{12}=-\frac{(N-1)}{N f}\left[f_{11}-2 \frac{N-2}{N-1} f_{12}-\frac{f_{22}}{N-1}\right] .
\end{aligned}
$$

For the Moran and Cannings process, the right members of eqs. A-43-A-44 are proportional to $1 / N$ and $(1-s)$, respectively.

\section{Stationary phenotypic distribution}

Here we derive the long-term stationary phenotypic distribution of $z$ by using the fixation probability of a single mutant (eq. A-35) in conjunction with the canonical diffusion of adaptive dynamics (Champagnat and Lambert, 2007).

\section{Canonical diffusion}

The so-called canonical diffusion (Champagnat and Lambert, 2007) is an equation describing the dynamics of the phenotype $z$ over evolutionary time in which the time to fixation is assumed to be a fast variable, so that fixation occurs instantly and the dynamics of $z$ over evolutionary time follows a stochastic trait substitution sequence. This canonical diffusion equation will allow us to derive the steady-state values of $z$. In order to use the canonical diffusion, we assume that with probability $\mu$ an individual mutates to a novel type and denote by $\sigma^{2}$ the variance of the mutation step $\delta$, which involves phenotypic deviations of order $\delta^{2}$. We also assume that the mutation mechanism is assumed here to be independent of the evolving phenotype $z$.

Then, as the mutation rate becomes very small $\mu \rightarrow 0$ and neglecting phenotypic deviation of order $\delta^{3}$, the dynamics of the phenotype of evolutionary time scale $t$ can be described 
by the stochastic differential equation

$$
\frac{\mathrm{d}}{\mathrm{d} t} z(t)=a(z(t))+\sqrt{b(z(t))} \frac{\mathrm{d} B(t)}{\mathrm{d} t},
$$

where $\mathrm{d} B(t) / \mathrm{d} t$ is the white noise (e.g., Karlin and Taylor, 1981, p. 342), and $a(z(t))$ and $b(z(t))$ are the infinitesimal mean and variance of the change in phenotype (Champagnat and Lambert, 2007, eq. 3). With our assumptions that the mutation mechanisms is independent of the evolving phenotype $z$ and that evolution occurs in a population of constant size $N$, we have from Champagnat and Lambert (2007, eq. 3) that

$$
\begin{aligned}
& a(z(t))=N \mu \sigma^{2} \dot{\pi}(z(t)) \\
& b(z(t))=\mu \sigma^{2},
\end{aligned}
$$

where the infinitesimal variance is independent of $z(t)$.

Eq. A-46 also provide the infinitesimal mean and variance of the diffusion process that arises as a solution of eq. A-45 (Karlin and Taylor, 1981, p. 376), and which describes the dynamics of the probability density $\psi(z, t)$ that phenotype $z$ is observed at time $t$ as

$$
\frac{\partial \psi(z, t)}{\partial t}=-\frac{\partial}{\partial z}[a(z) \psi(z, t)]+\frac{1}{2} \frac{\partial^{2}}{\partial z^{2}}[b(z) \psi(z, t)]
$$

which can also be derived by using the substitution rate approach of population genetics (Lehmann, 2012).

\section{Stationary distribution}

Our aim is to obtain the long term phenotypic distribution $\psi(z)=\lim _{t \rightarrow \infty} \psi(z, t)$, which is characterized by an evolutionary steady-state $\partial \psi(z, t) / \partial t=0$. In order to obtain this distribution, we first note that eq. A-47 can be expressed as $\partial \psi(z, t) / \partial t=-\partial J(z, t) / \partial z$, where $J(z, t)$ is the probability flux through $z$ at time $t$ (Kimura, 1964, p. 187;Gillespie, 1991, p. 157 ;Gardiner, 2004 , p. 119). At steady state, the probability flux $J(z)=\lim _{t \rightarrow \infty} J(z, t)$ is given by

$$
J(z)=a(z) \psi(z)-\frac{1}{2} \frac{\partial}{\partial z}[b(z) \psi(z)]
$$

Since we assume that the process takes place in some interval $[l, r]$, where $l$ and $r$ are, respectively, the left and right boundary of phenotypic values, there is no probability flux 
out of the interval $[l, r]$. We then have a process with reflecting boundaries (Gardiner, 2004, pp. 119-124), where $J(z)=0$ for any $z \in[l, r]$. The stationary distribution $\psi(z)$ is then obtained by solving the differential equation A-48, which gives

$$
\psi(z)=\frac{C}{b(z)} \exp \left[2 \int_{l}^{z} \frac{a(y)}{b(y)} \mathrm{d} y\right]
$$

where $C$ is a normalization constant (e.g., Kimura, 1964; Gillespie, 1991; Ewens, 2004; Gardiner, 2004). Substituting eq. A-46, we obtain

$$
\psi(z) \propto \exp \left[\int_{l}^{z} 2 N \dot{\pi}(y) \mathrm{d} y\right],
$$

which shows that the stationary distribution of the phenotypes will be independent of the features of the mutation process in the limit of a vanishing mutation rate.

\section{Example}

We will now evaluate $\psi(z)$ for the cooperator-defector game introduced in the main text (eq. 48). We will evaluate this for the Cannings process, as it allow use to cover both the Wright-Fisher and Moran model. For this model, we have from eq. 30 and that

$$
\dot{\pi}(z)=\frac{T_{2}}{N}(1-s) \tilde{w}_{i, i}
$$

where $T_{2}(1-s) / N=1 /(1+s)$ and $\tilde{w}_{i, i}$ is given by $(N-1)\left[f_{1}(z)-f_{2}(z) /(N-1)\right] /(N f)$ (from eq. A-17), whereby using eq. 48 gives

$$
\dot{\pi}(z)=\frac{(N-1)}{N(1+s) f}\left[b_{1}+4 b_{2} z-c_{1}-2 c_{2} z-\frac{b_{1}+4 b_{2} z}{N-1}\right] .
$$

Substituting eq. A-52 into eq. A-50, setting $l=0$ and using $s_{\mathrm{c}} \equiv 1 /(1+s)$ as the scaling factor between the various neutral processes (it can be checked that for the Moran process $s_{\mathrm{c}}=1 / 2$ and can thus be recovered when $s=1$ ), we obtain

$$
\begin{gathered}
\psi(z) \propto \exp \left[4 N s_{\mathrm{c}}\left(2 b_{2} c_{1}-b_{1} c_{2}\right)\left\{\tan ^{-1}\left(\frac{2 b_{1}-c_{1}}{B}\right)-\tan ^{-1}\left(\frac{2 b_{1}+8 b_{2} z-\mathrm{c} 1-2 c_{2} z}{B}\right)\right\}\right. \\
\left.+2 s_{\mathrm{c}} x\left(2 b_{2}(N-2)-c_{2} N+c_{2}\right)\left(\log \left[-z\left(2 b_{1}+4 b_{2} z-c_{1}-c_{2} z\right)-1\right]-i \pi\right)\right], \quad(\mathrm{A}-53)
\end{gathered}
$$

where $B=\sqrt{16 b_{2}-4 c_{2}-\left(c_{1}-2 b_{1}\right)^{2}}$. The function $\psi(z)$ can take a complex number but it takes a real number for every set of parameter values used in our simulation. 


\section{Individual based simulation}

Here, we present the algorithm of our individual based simulations. Each individual $i \in$ $\{1,2, \ldots, N\}$ can take a different phenotype $z_{i}(t) \in[0,1]$ at discrete time $t$. At each time step, each individual $i$ obtains fecundity $F_{i}(t)=\sum_{j \neq i} f\left(z_{i}(t), z_{j}(t)\right)$ from pairwise interactions with $N-1$ individuals. The next generation is then sampled according to the fecundity values of all individuals and according to the life-cycle assumption (Moran or Wright-Fisher). We applied the Moran or Wright-Fisher process exactly, which is different from Doebeli et al. (2004) and requires more computer time.

To introduce mutation, we assume that any newborn individual $k$ produced by an individual $i$ has phenotype $z_{k}(t+1)=z_{i}(t)$ with probability $1-\mu$ and phenotype $z_{k}(t+1)=z_{i}(t)+\delta$ with probability $\mu$. The mutation step size $\delta=\mathcal{N}(0, \sigma)$ is a random variable obeying the normal distribution with mean 0 and variance $\sigma^{2}$. When $z_{k}<0$ or $z_{k}>1$ occurs by mutation, we set $z_{k}=0$ or $z_{k}=1$ accordingly. When we choose a small $\sigma$ value, $\delta$ almost always takes a small value.

The initial condition is chosen so that all individuals have $z=0.1$ at $t=0$. When we calculate the stationary distribution, we run simulation until $t=T$ and the time-averaged frequencies over $[T / 2, T]$ are calculated.

\section{References}

Champagnat, N. and A. Lambert. 2007. Evolution of discrete populations and the canonical diffusion of adaptive dynamics. The annals of applied probability 17:102-155.

Doebeli, M., C. Hauert, and T. Killingback. 2004. The evolutionary origin of cooperators and defectors. Science 306:859-862.

Ewens, W. J. 2004. Mathematical Population Genetics. Springer-Verlag, New York.

Gardiner, C. W. 2004. Handbook of stochastic methods. Springer-Verlag, Berlin, 2th edn.

Gillespie, J. H. 1991. The Causes of Molecular Evolution. Oxford University Press, Oxford.

Karlin, S. and H. M. Taylor. 1981. A Second Course in Stochastic Processes. Academic Press, San Diego. 
Kimura, M. 1964. Diffusion Models in Population Genetics. Journal of applied probability $1: 177-232$.

Lehmann, L. 2012. The stationary distribution of a continuously varying strategy in a classstructured population under mutation-selection-drift balance. Journal of Evolutionary Biology 25:770-787.

Lehmann, L. and F. Rousset. 2009. Perturbation expansions of multilocus fixation probabilities for frequency-dependent selection with applications to the Hill-Robertson effect and to the joint evolution of helping and punishment. Theoretical Population Biology $76: 35-51$.

Rousset, F. 2004. Genetic Structure and Selection in Subdivided Populations. Princeton University Press, Princeton, NJ. 


\section{Corrigendum}

\section{Corrigendum to "Evolutionary and convergence stability for continuous phenotypes in finite populations derived from two-allele models"}

\section{[J. Theor. Biol. 310 (2012) 206-215]}

Joe Yuichiro Wakano ${ }^{\mathrm{a}, \mathrm{b}, *}$, Laurent Lehmann ${ }^{\mathrm{c}}$

a Meiji Institute for Advanced Study of Mathematical Sciences, Meiji University, Japan

${ }^{\mathrm{b}}$ PRESTO, Japan Science and Technology Agency, Japan

${ }^{\mathrm{c}}$ Department of Ecology and Evolution, University of Lausanne, Switzerland

In the above article the last equation in Eq. (38) and the same equation in Eq. (43) (both in page 211) were printed incorrectly. Here reproduced correctly

$$
Q_{C S}:\left.\frac{d w_{i, i}(z)}{d z}\right|_{z=z^{*}}=w_{i, i i}\left(z^{*}\right)+(N-1) w_{i, i j}\left(z^{*}\right)<0
$$

